ARTICLE

\title{
Mutant ASXL1 induces age-related expansion of phenotypic hematopoietic stem cells through activation of $\mathrm{Akt} / \mathrm{mTOR}$ pathway
}

Takeshi Fujino1, Susumu Goyama1, Yuki Sugiura², Daichi Inoue ${ }^{3,4}$, Shuhei Asada (1) 1,5, Satoshi Yamasaki ${ }^{6}$, Akiko Matsumoto ${ }^{6}$, Kiyoshi Yamaguchi", Yumiko Isobe ${ }^{7}$, Akiho Tsuchiya $^{1}$, Shiori Shikata ${ }^{1}$, Naru Sato ${ }^{1}$ Hironobu Morinaga ${ }^{8}$, Tomofusa Fukuyama (10 1, Yosuke Tanaka1, Tsuyoshi Fukushima', Reina Takeda', Keita Yamamoto ${ }^{1}$, Hiroaki Honda ${ }^{5}$, Emi K. Nishimura (1) ${ }^{8}$, Yoichi Furukawa ${ }^{7}$, Tatsuhiro Shibata ${ }^{6}$, Omar Abdel-Wahab (1) ${ }^{3}$, Makoto Suematsu (i) ${ }^{2} \&$ Toshio Kitamura (i) ${ }^{1 凶}$

Somatic mutations of ASXL1 are frequently detected in age-related clonal hematopoiesis $(\mathrm{CH})$. However, how ASXL1 mutations drive $\mathrm{CH}$ remains elusive. Using knockin (KI) mice expressing a C-terminally truncated form of ASXL1-mutant (ASXL1-MT), we examined the influence of ASXL1-MT on physiological aging in hematopoietic stem cells (HSCs). HSCs expressing ASXL1-MT display competitive disadvantage after transplantation. Nevertheless, in genetic mosaic mouse model, they acquire clonal advantage during aging, recapitulating $\mathrm{CH}$ in humans. Mechanistically, ASXL1-MT cooperates with BAP1 to deubiquitinate and activate AKT. Overactive Akt/mTOR signaling induced by ASXL1-MT results in aberrant proliferation and dysfunction of HSCs associated with age-related accumulation of DNA damage. Treatment with an mTOR inhibitor rapamycin ameliorates aberrant expansion of the HSC compartment as well as dysregulated hematopoiesis in aged ASXL1-MT KI mice. Our findings suggest that ASXL1-MT provokes dysfunction of HSCs, whereas it confers clonal advantage on HSCs over time, leading to the development of $\mathrm{CH}$.

\footnotetext{
${ }^{1}$ Division of Cellular Therapy, The Institute of Medical Science, The University of Tokyo, Minato-ku, Tokyo, Japan. ${ }^{2}$ Department of Biochemistry, Keio University School of Medicine, and Japan Science and Technology Agency (JST), Exploratory Research for Advanced Technology (ERATO), Suematsu Gas Biology Project, Shinjuku-ku, Tokyo, Japan. ${ }^{3}$ Human Oncology and Pathogenesis Program, Memorial Sloan-Kettering Cancer Center and Weill Cornell Medical College, New York, USA. ${ }^{4}$ Department of Hematology-Oncology, Institute of Biomedical Research and Innovation, Foundation for Biomedical Research and Innovation at Kobe, Kobe City, Hyogo, Japan. ${ }^{5}$ Field of Human Disease Models, Major in Advanced Life Sciences and Medicine, Tokyo Women's Medical University, Shinjuku-ku, Tokyo, Japan. ${ }^{6}$ Laboratory of Molecular Medicine, Human Genome Center, The Institute of Medical Science, The University of Tokyo, Minato-ku, Tokyo, Japan. ${ }^{7}$ Division of Clinical Genome Research, Advanced Clinical Research Center, The Institute of Medical Science, The University of Tokyo, Minato-ku, Tokyo, Japan. ${ }^{8}$ Department of Stem Cell Biology, Medical Research Institute, Tokyo Medical and Dental University,

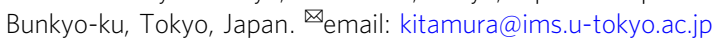


B illions of blood cells are produced daily to maintain homeostasis in a human body. Hematopoietic stem cells (HSCs) are a rare population, but they are capable of selfrenewal and multi-lineage differentiation, which play a pivotal role in maintaining life-long hematopoiesis. In the aging process, HSCs are subject to both cell-intrinsic and cell-extrinsic stresses, which may result in cellular senescence and increased incidence of malignancy ${ }^{1-3}$. It has been reported that aging is associated with a significant expansion in surface marker-defined immunophenotypic HSCs (pHSCs), while these cells reduce repopulation ability ${ }^{4-7}$. Progenitors with limited differentiation potential and impaired repopulation ability accumulate within the pHSC compartment, which may serve as the mechanistic basis for this phenomenon $^{5-9}$. Such pHSCs may also contribute to the development of clonal hematopoiesis $(\mathrm{CH})$ or clonal hematopoietic disease, but this concept has yet to be validated ${ }^{1,9}$.

$A S X L 1$, a gene frequently mutated in $\mathrm{CH}$, is one of three human homologs of the Drosophila Asx gene and is involved in epigenetic regulation $^{10-13}$. In addition to $\mathrm{CH}$, somatic mutations of $A S X L 1$ gene are detected in myeloid neoplasms including myelodysplastic syndromes (MDS), chronic myelomonocytic leukemia (CMML), and acute myeloid leukemia (AML) ${ }^{14-18}$. Most ASXL1 mutations detected in $\mathrm{CH}$ and hematological malignancies are frameshift or nonsense mutations in the last exon, generating a C-terminally truncated form of ASXL1 14,15,17-21. Such truncated ASXL1 proteins are indeed expressed in leukemic cells and likely confer change-of-function ${ }^{22}$. Recently, we and others have shown that the mutant ASXL1 interacts with a deubiquitinase BAP1 to form the Polycomb-repressive deubiquitinase (PR-DUB) complex, which efficiently deubiquitinates H2AK119Ub ${ }^{23,24}$. The hyperactive PR-DUB complex of mutant ASXL1 and BAP1 upregulates several target genes, including $H O X$ genes, by deubiquitinating $\mathrm{H} 2 \mathrm{AK} 119 \mathrm{Ub}$ to promote myeloid leukemogenesis ${ }^{23}$. In hematopoietic lineage-specific conditional knockin (KI) mice expressing a C-terminally truncated form of mutant ASXL1 (ASXL1-MT; 1900-1922del;E635RfsX15) ${ }^{25}$, global reduction was observed in H3K4me3 and H2AK119Ub. In particular, levels of H3K4me3 at the loci of erythroid differentiation-related genes, such as Id3 and Sox6, were markedly decreased, suggesting that ASXL1-MT impairs hematopoiesis through dysregulated epigenetic modifications.

It is now generally recognized that cells that are well-adapted to selective pressures acquire a fitness advantage, leading to clonal expansion during aging ${ }^{26-29}$. Clonal expansion of such blood cells harboring somatic mutations is termed $\mathrm{CH}$. $\mathrm{CH}$ is commonly observed in elderly individuals in the absence of apparent hematological abnormalities ${ }^{19-21}$. Despite normal hematological parameters, individuals with $\mathrm{CH}$ are at increased risk of hematological malignancies, indicating that $\mathrm{CH}$ can be a preleukemic condition. Mutations most commonly detected in $\mathrm{CH}$ include the epigenetic regulators DNMT3A, TET2, and ASXL1. From the results of transplantation experiments using Tet2-deficient or Dnmt3a-deficient mice, the increased self-renewal of HSCs could explain why loss-of-function mutations of DNMT3A and TET2 are frequently detected in $\mathrm{CH}^{30-33}$. On the other hand, several recent studies have shown reduced numbers and functions of HSCs in mutant ASXL1 KI mice $25,34,35$. Thus, identifying how ASXL1 mutations promote the development of $\mathrm{CH}$ has remained an unsolved issue.

In the present study, we examined the influence of ASXL1-MT on physiological aging using ASXL1-MT KI mice. We found that ASXL1-MT confers a competitive disadvantage on HSCs after transplantation. On the other hand, in genetic mosaic mouse model, we observed stronger growth advantage in LT-HSCs expressing ASXL1-MT specifically in native hematopoiesis, recapitulating $\mathrm{CH}$ in humans. As for the molecular mechanisms by which ASXL1-MT induces $\mathrm{CH}$, we show that ASXL1-MT binds and activates Akt by stabilizing phosphorylated Akt in concert with Bap1. Activation of Akt/mTOR pathway induced by ASXL1-MT causes aberrant cell cycle progression and proliferation in the HSC compartment. At the same time, it also provokes dysfunction of HSCs associated with mitochondrial activation, elevated ROS levels, and increased DNA damage. These molecular changes can cause $\mathrm{CH}$ with increased risk of leukemogenesis, which can be ameliorated by rapamycin treatment.

\section{Results}

ASXL1-MT reduces the number and function of HSPCs in young mice. To study the effects of ASXL1-MT on hematopoiesis, we crossbred ASXL1-MT KI mice with Vav-Cre transgenic mice. Consistent with our previous reports ${ }^{25}$, young Vav-Cre ASXL1MT KI mice did not show significant changes in hematological parameters (hereinafter, all experiments were performed with 6-12-week-old for young mice) (Fig. 1a). We also observed no significant changes in the frequency of myeloid cells $\left(\mathrm{CD} 11 \mathrm{~b}^{+}\right), \mathrm{B}$ cells $\left(\mathrm{B}_{22}{ }^{+}\right)$, and $\mathrm{T}$ cells $\left(\mathrm{CD}^{+}\right)$between control and Vav-Cre ASXL1-MT KI mice (Fig. 1b). Young Vav-Cre ASXL1-MT KI mice showed normal bone marrow cellularity (Fig. 1c), but exhibited a significant decrease in the frequency of hematopoietic stem and progenitor cells (HSPCs), including $\mathrm{Lin}^{-} \mathrm{Scal}^{+} \mathrm{c}$-kit ${ }^{+}$ (LSK) cells, multipotent progenitors (MPPs; CD $\left.48^{+} \mathrm{CD} 150^{-} \mathrm{LSK}\right)$ and long-term HSCs (LT-HSCs; CD48 ${ }^{-} \mathrm{CD} 150^{+}$LSK) (Fig. 1d and Supplementary Fig. 1). Competitive transplantation assays using whole bone marrow cells or defined populations (MPPs and LT-HSCs) revealed impaired repopulation ability and skewed differentiation potential toward myeloid lineage in ASXL1-MT KI HSPCs (Fig. 1e, f, Supplementary Fig. 2a-d). Thus, consistent with the previous reports, ASXL1-MT reduced the number and function of HSPCs in young mice.

To characterize the defective HSPC function of young $\mathrm{Vav}$-Cre ASXL1-MT KI mice, we evaluated apoptosis and cell cycle status. The frequency of Annexin V-positive cells was significantly increased in HSPCs from Vav-Cre ASXL1-MT KI mice (Fig. 1g), indicating that ASXL1-MT KI HSPCs are more prone to apoptosis than are normal HSPCs. Ki-67/DAPI staining revealed a significantly lower frequency of LT-HSCs in G0 phase, and a higher frequency of both MPPs and LT-HSCs in S/G2/M phase in Vav-Cre ASXL1-MT KI mice (Fig. 1h). These data suggest that increased apoptosis and loss of quiescence contribute to the defective repopulation ability in HSPCs of young Vav-Cre ASXL1-MT KI mice.

ASXL1-MT confers a clonal advantage on LT-HSCs specifically in native hematopoiesis. Next, we assessed age-associated changes in hematopoiesis driven by ASXL1-MT. Analyses of peripheral blood cells showed mild leukocytopenia and mild anemia, which are prominent when compared with age-matched control mice, as well as moderate thrombocytosis in aged Vav-Cre ASXL1-MT KI mice (hereinafter, all experiments were performed with 20-24-month-old for aged mice) (Fig. 2a). Aged Vav-Cre ASXL1-MT KI mice exhibited a myeloid-biased hematopoiesis and hypocellular bone marrow, suggesting dysfunction of hematopoiesis (Figs. 2b, 9d, and e). Aged Vav-Cre ASXL1-MT KI mice do not develop apparent hematological diseases and their lifetime is equal to wild-type mice. Interestingly, the frequencies of LT-HSCs were markedly increased in aged Vav-Cre ASXL1MT KI mice (Fig. 2c). We then assessed the functions of LT-HSCs in aged Vav-Cre ASXL1-MT KI mice. Single-cell liquid cultures revealed a decrease in the frequency of colony-forming LT-HSC from aged Vav-Cre ASXL1-MT KI mice compared with age-matched control mice (Supplementary Fig. 3a and b). 

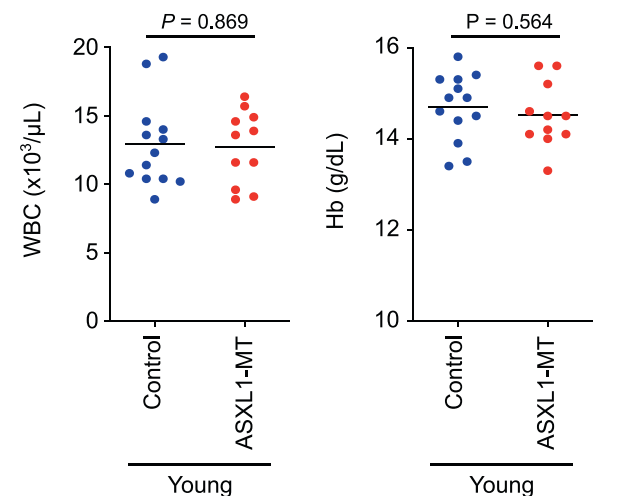

d

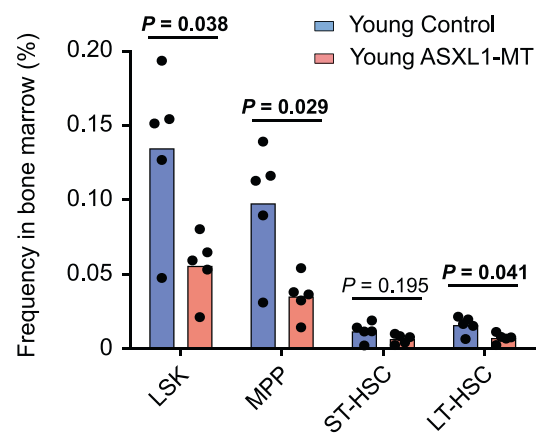

$\mathbf{f}$

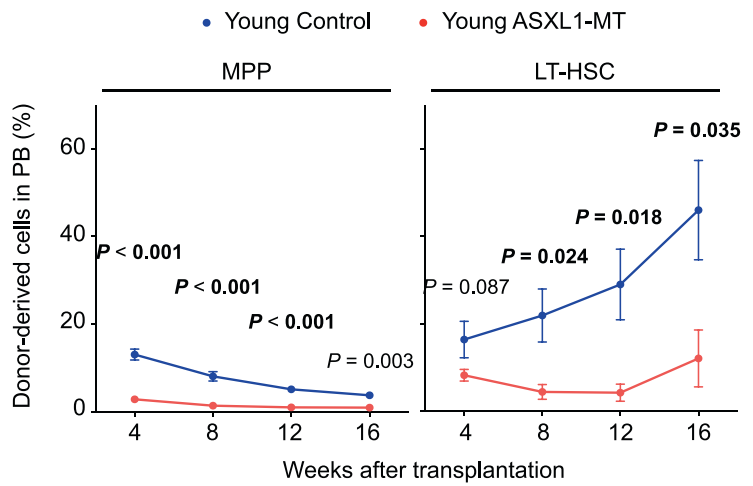

b

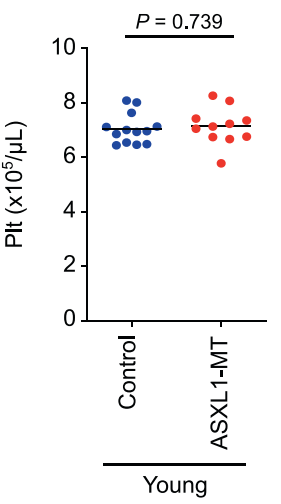

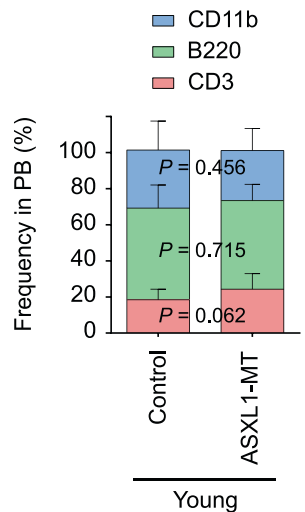

C

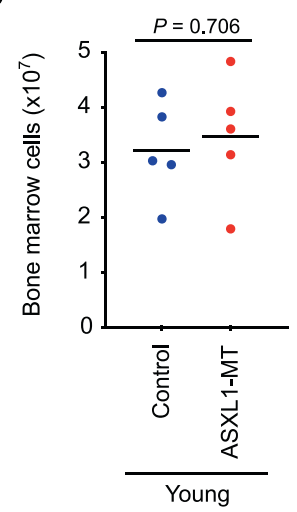

e

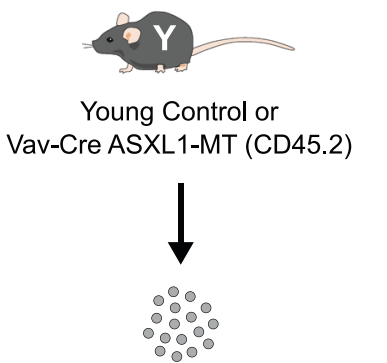

3000 MPPs or 200 LT-HSCs

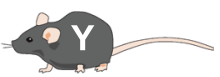

WT competitor (CD45.1)

\section{$4 \times 10^{5}$ whole BM cells}

\section{g}

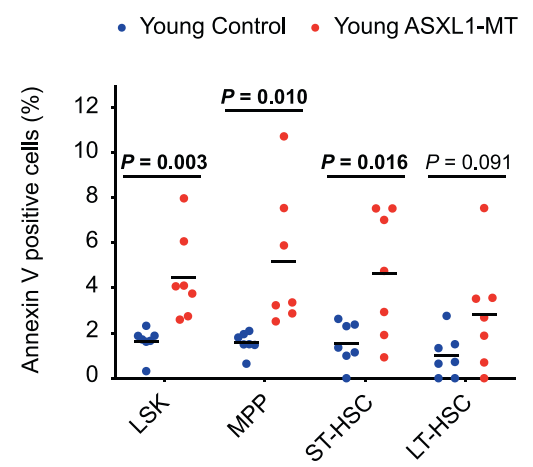

h

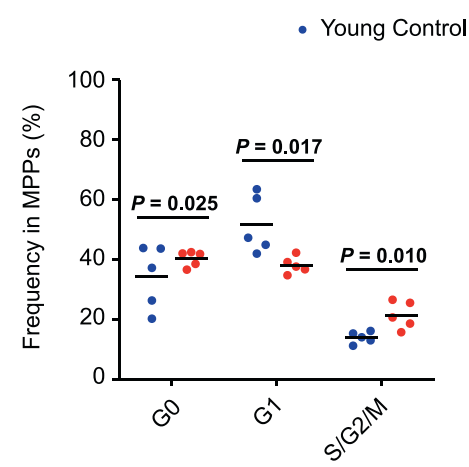

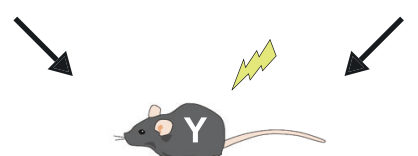

Lethally irradiated WT recipient (CD45.1)

Chimerism analysis

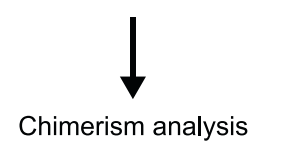

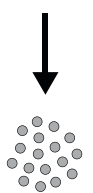

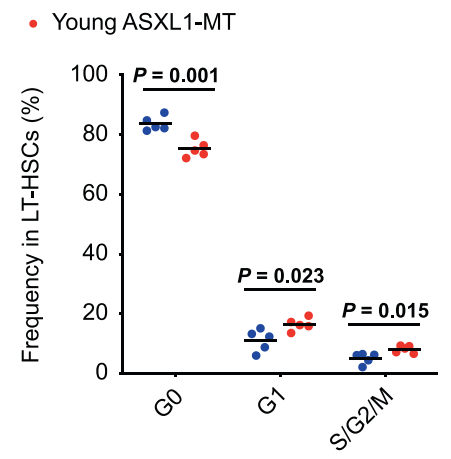

Furthermore, competitive transplantation assays using LT-HSCs from aged Vav-Cre ASXL1-MT KI mice and age-matched control mice showed that aged LT-HSCs expressing ASXL1-MT exhibited markedly compromised production of peripheral blood and tended to exhibit impaired regeneration of bone marrow cells including LT-HSCs (Fig. 2d-f). We also performed homing assays using LSK cells from both young and aged Vav-Cre ASXL1-MT KI mice. These analyses revealed that LSK cells from aged Vav-Cre ASXL1-MT KI mice exhibited a significant decrease in homing capability compared to age-matched control mice in addition to intrinsic defects detected by in vitro colony forming assays (Supplementary Fig. 3a-d). Thus, the increased LT-HSCs 
Fig. 1 ASXL1-MT causes dysfunction of HSPCs associated with increased apoptosis and altered cell cycle status. a Enumeration of white blood cells $(\mathrm{WBC})$, hemoglobin $(\mathrm{Hb})$, and platelets (PIt) in peripheral blood of young Vav-Cre ASXL1-MT KI mice $(n=13$ (Control), 11 (ASXL1-MT)). b Frequency of myeloid cells $\left(\mathrm{CD}_{11} \mathrm{~b}^{+}\right), \mathrm{B}$ cells $\left(\mathrm{B} 22 \mathrm{O}^{+}\right)$, and T cells $\left(\mathrm{CD} 3^{+}\right)$in peripheral white blood cells of young Vav-Cre ASXL1-MT KI mice $(n=13(\mathrm{Control})$, 11 (ASXL1-MT)). c Absolute numbers of bone marrow cells per leg in young Vav-Cre ASXL1-MT KI mice $(n=5)$. d Frequency of LSK cells, multipotent progenitors (MPPs), short-term HSCs (ST-HSCs) and long-term HSCs (LT-HSCs) in bone marrow cells of young Vav-Cre ASXL1-MT KI mice ( $n=5$ ). e The experimental design for competitive transplantation assays. 3000 MPPs or 200 LT-HSCs isolated from young control or young Vav-Cre ASXL1-MT KI mice were transplanted into lethally irradiated recipient mice with $4 \times 10^{5}$ whole bone marrow cells. $\mathbf{f}$ Levels of donor chimerism in peripheral blood were analyzed at the indicated weeks after transplantation ( $n=3$ (Control), 4 (ASXL1-MT)). Data are mean \pm s.e.m. $\mathbf{g}$ Apoptosis analysis of HSPCs of young Vav-Cre ASXL1-MT KI mice $(n=7)$. h Cell cycle analysis with Ki-67/DAPI staining of MPPs (left panel) and LT-HSCs (right panel) of young Vav-Cre ASXL1MT KI mice $(n=5)$. Data are mean \pm s.d. unless otherwise noted. ${ }^{\star} P \leq 0.05,{ }^{\star \star} P \leq 0.01,{ }^{\star \star \star} P \leq 0.001$; two-tailed Student's $t$-test.

in aged Vav-Cre ASXL1-MT KI mice are not functional HSCs with long-term repopulation potential (hereinafter, referred to as "immunophenotypic LT-HSCs (pLT-HSCs)").

It appears that ASXL1-MT promotes age-related changes in the hematopoietic system of Vav-Cre ASXL1-MT KI mice (e.g. anemia, myeloid-skewed differentiation, and hypocellular bone marrow). Therefore, we next evaluated the frequency of CD41positive pLT-HSCs, which has been reported to skew differentiation toward myeloid and megakaryocytic lineages, and to expand during aging within the pLT-HSC compartment ${ }^{8,9,36,37}$. This analysis revealed that most pLT-HSCs were positive for CD41 in aged Vav-Cre ASXL1-MT KI mice, while $50-70 \%$ were positive in age-matched control mice (Supplementary Fig. 3e). These data imply that ASXL1-MT accelerates the physiological aging of LTHSCs.

Next, to assess the competitive growth of ASXL1-MT KI cells in native hematopoiesis without transplantation, we bred ASXL1-MT KI mice with Mx1-Cre transgenic mice and partially induced expression of ASXL1-MT by polyinosine-polycytidine (pIpC) injections (Fig. 3a). As these mice carry the floxed allele of ASXL1MT-IRES-GFP, cells expressing ASXL1-MT also express GFP (Supplementary Fig. 1). In these genetic mosaic mice, frequency of ASXL1-MT expressing cells (GFP positive) gradually increased in peripheral blood (Fig. 3b). Similar to the phenotypes of aged VavCre ASXL1-MT KI mice, expression of ASXL1-MT in this model also caused anemia, thrombocytosis, and myeloid-biased differentiation with age (Fig. $3 c$ and Supplementary Fig. $4 a-d)$. The frequency of LT-HSCs expressing ASXL1-MT was increased in aged Mx1-Cre ASXL1-MT KI mice along with progression of cell cycle (Fig. 3d and e). Eventually, LT-HSCs expressing ASXL1-MT occupied bone marrow to outcompete their normal counterpart 2 years after $\mathrm{pIpC}$ injections, recapitulating $\mathrm{CH}$ in humans (Fig. 3f). Given that levels of pro-inflammatory cytokines including TNF- $\alpha$ and IFN- $\gamma$ were similar in aged control and aged Mx1-Cre ASXL1-MT KI mice (Supplementary Fig. 4e and f), these phenotypic changes were caused by ASXL1-MT itself, not by the inflammation-induced $M x 1-C r e$ expression.

Thus, ASXL1-MT causes a competitive disadvantage of LTHSCs during transplantation; nevertheless, it confers a clonal advantage on LT-HSCs in native hematopoiesis. To investigate cell intrinsic and extrinsic mechanisms that mediate the effect of ASXL1-MT on HSC function, we performed transplantation assays with three different settings (Supplementary Fig. 5a, d, and f). First, we transplanted bone marrow cells from young Mx1-Cre ASXL1-MT KI mice into wild-type recipient mice and induced ASXL1-MT expression by pIpC injections one month after transplantation. Induction of ASXL1-MT after engraftment did not lower chimerism of peripheral blood but rather tended to increase the frequency of donor-derived bone marrow cells, suggesting that ASXL1-MT exerts competitive disadvantage on LT-HSCs, specifically during recovery from transplantation (Supplementary Fig. 5a-c). Second, we transplanted bone marrow cells from young Vav-Cre ASXL1-MT KI mice into young or aged wild-type recipient mice. The frequencies of ASXL1-MTexpressing cells were similar regardless of the age of recipient mice (Supplementary Fig. 5d and e). Finally, we transplanted bone marrow cells from young $\mathrm{Vav}$-Cre ASXL1-MT KI mice into Mx1-Cre ASXL1-MT KI mice that had been induced expression of ASXL1-MT by pIpC injections one month before transplantation. Expression of ASXL1-MT in non-hematopoietic cells of the recipient bone marrow did not affect the chimerism of ASXL1MT-expressing cells in peripheral blood (Supplementary Fig. $5 f$ and g). Taken together, we concluded that expression of ASXL1MT in LT-HSCs promotes the age-related clonal expansion independent of either aging of microenvironment or ASXL-MTexpressing stromal cells in native hematopoiesis.

ASXL1-MT induces aberrant expansion of the LT-HSC compartment through activation of Akt/mTOR pathway. To gain insight into the mechanism underlying the growth advantage of pLT-HSCs expressing ASXL1-MT, we performed RNA-seq analysis using HSPCs from young Vav-Cre ASXL1-MT KI mice and littermate control mice. Geneset enrichment analysis (GSEA) suggested that Akt/mTOR pathway was activated in ASXL1-MT KI HSPCs (Fig. 4a). Consistent with the results of GSEA, intracellular flow cytometry analyses revealed that phosphorylation of Akt and S6, which indicates activation of Akt and mTORC1, respectively, were elevated in young ASXL1-MT KI HSPCs (Fig. 4b, c, Supplementary Fig. 6a, b). Next, we evaluated the age-related change of Akt/mTOR activity in Vav-Cre ASXL1-MT KI mice. LT-HSCs of both young and aged mice exhibited activation of Akt/mTOR signaling to the same degree in Vav-Cre ASXL1-MT KI mice compared with age-matched control mice (Fig. $4 \mathrm{~d}$ and e).

As Akt/mTOR signaling positively controls cell proliferation, we inferred that aberrant proliferation of ASXL1-MT-expressing pLTHSCs was caused by enhanced Akt/mTOR signaling. Therefore, we treated aged Vav-Cre ASXL1-MT KI mice and age-matched control mice with an mTOR inhibitor rapamycin for 8 weeks (Fig. 5a). Intriguingly, treatment with rapamycin reduced the frequency of pLT-HSCs in aged Vav-Cre ASXL1-MT KI mice (Fig. 5b and c). Similar to young mice, pLT-HSCs in G0 phase were significantly decreased in aged Vav-Cre ASXL1-MT KI mice, which was normalized by rapamycin treatment (Fig. 5d). In contrast, ASXL1-MT had no effect on the frequency of apoptotic LT-HSCs in aged mice (Fig. 5e). Treatment with an Akt inhibitor perifosine also normalized cell cycle status in aged Vav-Cre ASXL1-MT KI mice (Fig. $5 \mathrm{f}$ and g). These data suggest that abnormal cell cycle progression contributes to gradual expansion of the LT-HSC compartment during aging in Vav-Cre ASXL1-MT KI mice. Collectively, the activated Akt/mTOR pathway plays a pivotal role to confer growth advantage on LT-HSCs expressing ASXL1-MT.

ASXL1-MT/BAP1 complex promotes AKT deubiquitination and stabilization. Previous studies have shown that phosphorylated $\mathrm{AKT}$ is ubiquitinated and degraded ${ }^{38-42}$, and wild-type 
a

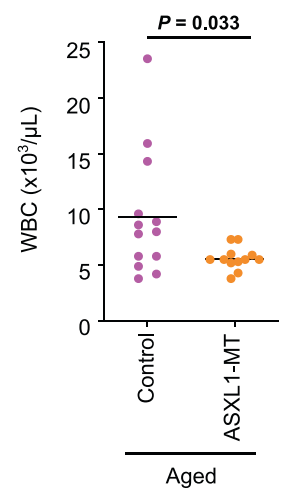

c

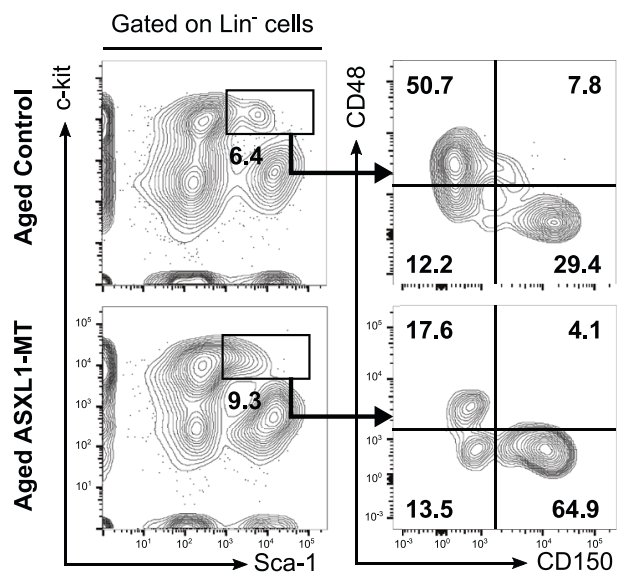

d

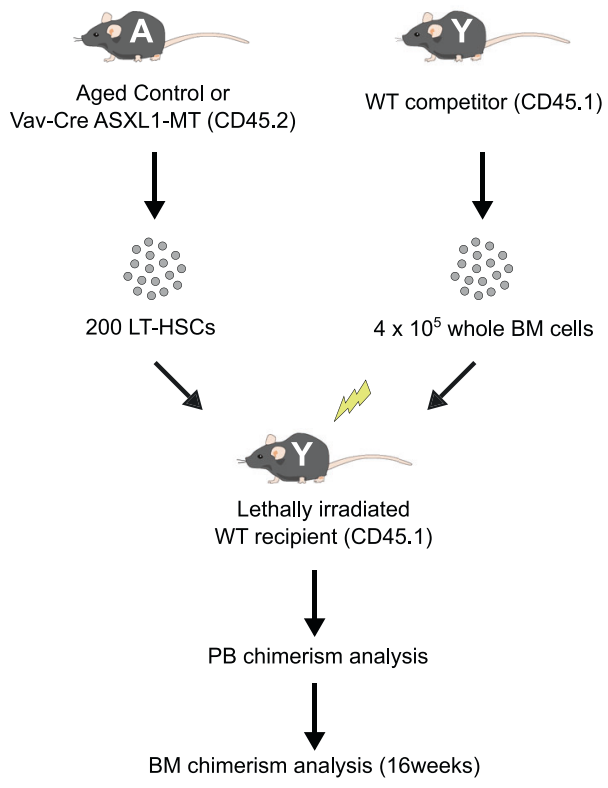

b
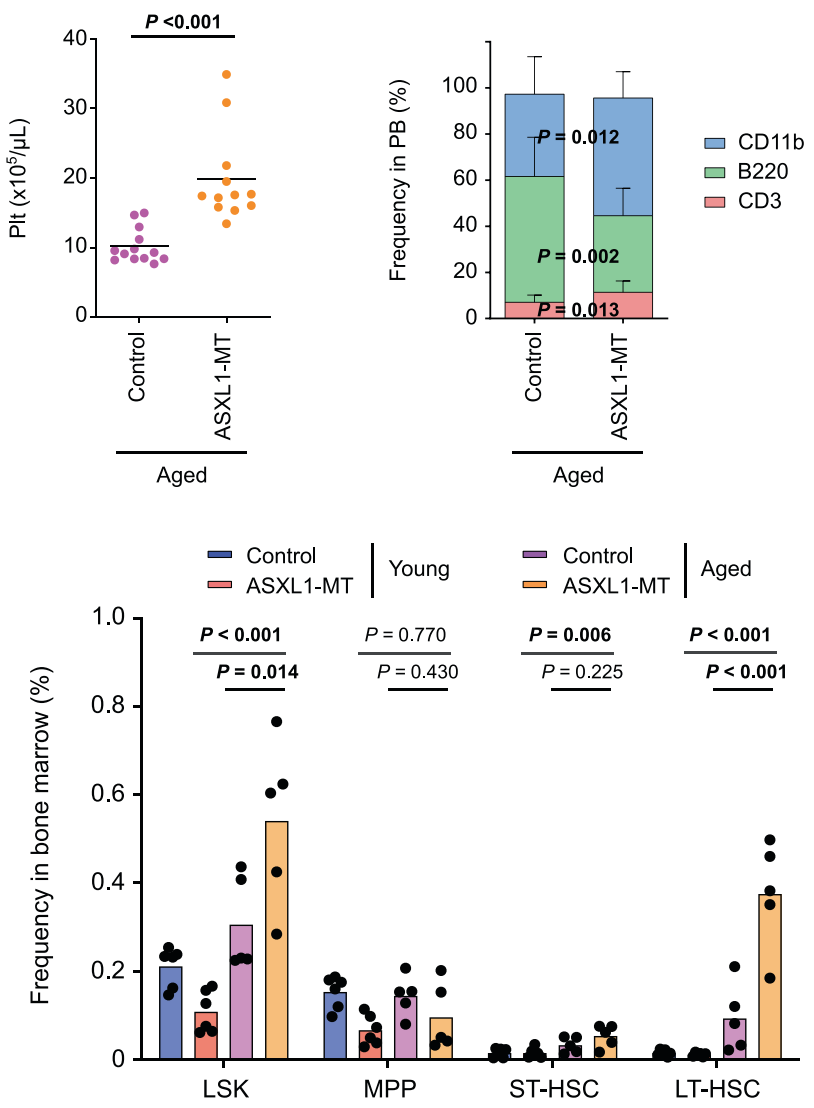

e

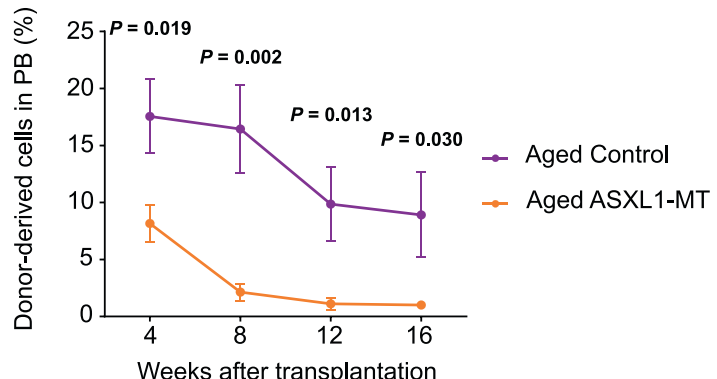

f

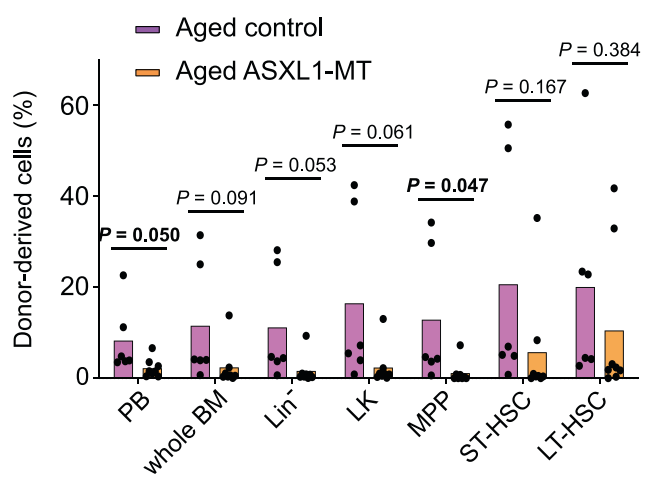

and Supplementary Fig. 7a). Because mutant ASXL1 is known to stabilize BAP1 and enhance its DUB activity ${ }^{23,24}$, we next examined whether ubiquitination of $\mathrm{AKT}$ is removed by the ASXL1-MT/BAP1 complex. Consistent with our previous reports $^{23}$, ASXL1-MT and BAP1 were mutually stabilized 
Fig. 2 ASXL1-MT expands the pLT-HSC compartment along with dysregulated hematopoiesis during aging. a Enumeration of white blood cells (WBC), hemoglobin $(\mathrm{Hb})$, and platelets (PIt) in peripheral blood of aged Vav-Cre ASXL1-MT KI mice $(n=13$ (Control) and 12 (ASXL1-MT)). b The frequency of myeloid cells $\left(\mathrm{CD}_{11} \mathrm{~b}^{+}\right), \mathrm{B}$ cells $\left(\mathrm{B} 22 \mathrm{O}^{+}\right)$, and T cells $\left(\mathrm{CD}^{+}\right)$in peripheral white blood cells of aged Vav-Cre ASXL1-MT KI mice $(n=13(\mathrm{Control})$ and 12 (ASXL1-MT)). c The frequency of LSK cells, MPPs, ST-HSCs, and LT-HSCs in bone marrow cells of young and aged Vav-Cre ASXL1-MT KI mice ( $n=6$ (Young) and 5 (Aged)). Representative FACS plot (left panel) and summarized data (right panel) are shown. $\mathbf{d}$ The experimental design for competitive transplantations. 200 LT-HSCs isolated from aged Vav-Cre ASXL1-MT KI mice and age-matched control mice were transplanted into lethally irradiated recipient mice with $4 \times 10^{5}$ whole bone marrow cells. e Levels of donor chimerism in peripheral blood were analyzed at the indicated weeks after transplantation $(n=7$ (Control) and 8 (ASXL1-MT)). $\mathbf{f} 6$ months after transplantation, the frequency of donor-derived cells in peripheral blood (PB), whole bone marrow cells, Lin ${ }^{-}$cells, Lin ${ }^{-}$c-kit $^{+}$Sca1-(LK) cells, MPPs, ST-HSCs, and LT-HSCs were analyzed $(n=6$ (Control) and 8 (ASXL1-MT)). Data are mean \pm s.e.m. Data are mean \pm s.d. unless otherwise noted. Data are assessed by two-tailed Student's $t$-test (a, b, e, f) or one-way ANOVA with Tukey-Kramer's post-hoc test $(\mathbf{c}) .{ }^{\star} P \leq 0.05,{ }^{\star \star} P \leq 0.01,{ }^{\star \star \star} P \leq 0.001$

(Fig. 6c). Notably, expression of BAP1 together with ASXL1-MT, but not with wild-type ASXL1, effectively deubiquitinated AKT mainly through K48-linked ubiquitin (Fig. 6c, d, and Supplementary Fig. 7b). Thus, these data suggest that AKT is a nonhistone targets of the ASXL1-MT/BAP1 DUB complex.

To determine the role of endogenous Bap1 on Akt signaling, we then assessed the effect of Bap1 deletion in murine bone marrow cells transformed by combined expression of SETBP1D868N and ASXL1-MT (cSAM cells: cells with combined expressing of mutant SETBP1 and ASXL1-MT) in which Akt is activated $^{45}$. As described in our previous report ${ }^{23}$, Bap1 depletion destabilized and reduced ASXL1-MT protein levels (Fig. 6e). In addition, Bap1 deletion in cSAM cells decreased expression of phosphorylated Akt (Fig. 6e). A time course experiment with IL-3 stimulation revealed attenuated and shortened phosphorylation of Akt in Bap1-depleted cSAM cells (Fig. $6 \mathrm{f}$ and g). These data suggest that ASXL1-MT cooperates with Bap1 to deubiquitinate and stabilize phosphorylated Akt, leading to activation of Akt/mTOR pathway.

ASXL1-MT activates mitochondrial metabolism and alters mitochondrial dynamics. GSEA of the RNA-seq data also suggested the aberrant activation of mitochondrial metabolism in HSPCs of Vav-Cre ASXL1-MT KI mice (Fig. 7a, Supplementary Fig. 8a, and Supplementary Table 1). As recent studies have shown that mitochondrial dynamics are closely linked to stem cell function $^{46-48}$, we therefore examined the possible influence of ASXL1-MT on mitochondrial activity. MitoTracker staining showed increased mitochondrial membrane potential in HSPCs of $\mathrm{Vav}$-Cre ASXL1-MT KI mice (Fig. 7b). In addition, extracellular flux analyses revealed increased oxygen consumption rates (OCR) in c-kit ${ }^{+}$cells of Vav-Cre ASXL1-MT KI mice (Fig. 7c). We then conducted ion chromatography-mass spectrometry (IC-MS)-based metabolomics to analyze intracellular metabolites using HSPCs. As shown in Supplementary Fig. 8b and $c$, the pool of TCA cycle-intermediates as well as levels of ATP were increased in HSPCs of Vav-Cre ASXL1-MT KI mice. These data suggest enhanced mitochondrial respiration in HSPCs of $\mathrm{Vav}$-Cre ASXL1-MT KI mice. Interestingly, immunofluorescence staining of the mitochondrial protein Tom20 revealed that mitochondria in HSPCs of Vav-Cre ASXL1-MT KI mice tended to form a few large aggregates, while those in HSPCs of control mice showed a more punctuated and dispersed morphology (Fig. 7d and e). These morphological changes suggest altered mitochondrial fission and fusion in HSPCs of Vav-Cre ASXL1-MT KI mice $47,49,50$. Collectively, these results indicate that ASXL1-MT enhances mitochondrial activity and alters mitochondrial dynamics in HSPCs.

ROS-mediated DNA damage causes dysfunction of HSPCs in ASXL1-MT KI mice. Mitochondria are the major source of ROS, which can cause DNA damage ${ }^{51,52}$. We therefore assessed the levels of ROS in Vav-Cre ASXL1-MT KI mice. As expected, ASXL1-MT KI HSPCs showed increased ROS levels (Fig. 7f). Moreover, alkaline comet assay using pLT-HSCs revealed increased DNA strand breaks in young Vav-Cre ASXL1-MT KI mice compared to young control mice (Fig. 7g). We also observed increased nuclear foci of $\gamma$-H2AX in ASXL1-MT KI HSPCs after transplantation (Supplementary Fig. 8d). Notably, aged Vav-Cre ASXL1-MT KI mice harbor more DNA damage than do young Vav-Cre ASXL1-MT KI mice and age-matched control mice (Fig. $7 \mathrm{~g}$ ), implicating stronger age-related accumulation of DNA damage in pLT-HSCs of Vav-Cre ASXL1-MT KI mice.

Next, we investigated whether treatment with the antioxidant scavenger $\mathrm{N}$-acetylcysteine (NAC) mitigates DNA damage and restores the impaired repopulation potential of HSPCs in Vav-Cre ASXL1-MT KI mice. We transplanted bone marrow cells from Vav-Cre ASXL1-MT KI mice or littermate control mice, treated with NAC for 8 weeks, into recipient mice (Fig. 8a). NAC treatment effectively normalized ROS and $\gamma$-H2AX levels of ASXL1-MT KI HSPCs (Fig. 8b and c), and partially restored their repopulation ability (Fig. 8d). Combined overexpression of catalase and a ROS-detoxifying enzyme, manganese superoxide dismutase (Cat/SOD2), also rescued the defective repopulation potential of HSPCs of Vav-Cre ASXL1-MT mice (Fig. 8f and g). Thus, reduction of ROS efficiently reverts the dysfunction of HSPCs in Vav-Cre ASXL1-MT KI mice.

p53 serves to maintain genomic stability through DNA damage response and regulation of ROS levels, but it can also cause dysfunction of $\mathrm{HSCs}^{53-55}$. To assess the influence of p53 on ASXL1-MT KI HSPCs, we crossed Vav-Cre ASXL1-MT KI mice with $p 53^{-/-}$mice. ASXL1-MT KI/p53-/- mice showed significantly higher frequency of LT-HSCs than ASXL1-MT KI mice (Supplementary Fig. 9a and b). Competitive transplantation assays revealed that loss of $p 53$ restored the repopulation potential of ASXL1-MT KI HSPCs (Supplementary Fig. 9c). Meanwhile, ASXL1-MT KI/p53-/- mice are prone to early onset of thymic lymphomas compared with $p 53^{-/-}$mice (Supplementary Fig. 9d-f). Transduction of dominant-negative form of p53 (p53DD) increased the repopulation potential of ASXL1-MT KI HSPCs associated with increased ROS levels and DNA damage (Supplementary Fig. $9 \mathrm{~g}-\mathrm{j}$ ). These findings indicate that p53 plays a critical role in suppressing ROS levels and preventing tumor formation at the expense of HSPC functions in Vav-Cre ASXL1MT KI mice, presumably by eradicating cells with critical DNA damage.

Overactive Akt/mTOR signaling is responsible for dysregulated hematopoiesis in aged ASXL1-MT KI mice. As mTORC1, a major downstream effector molecule of AKT signaling, regulates mitochondrial biogenesis $46,56,57$, we inferred that the elevated mitochondrial activity was caused by enhanced Akt/mTOR signaling. Consistent with this hypothesis, treatment with 


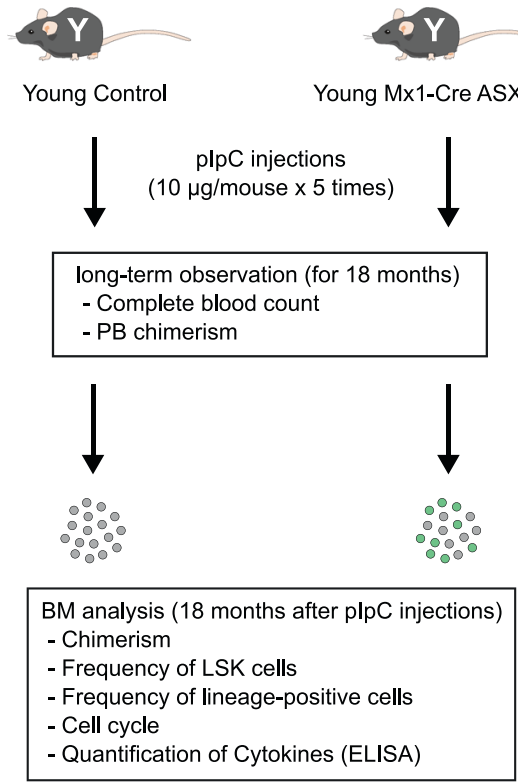

d

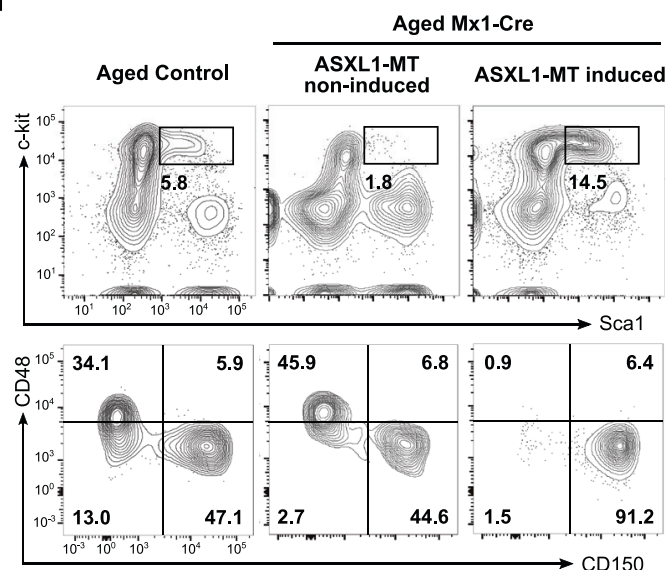

e

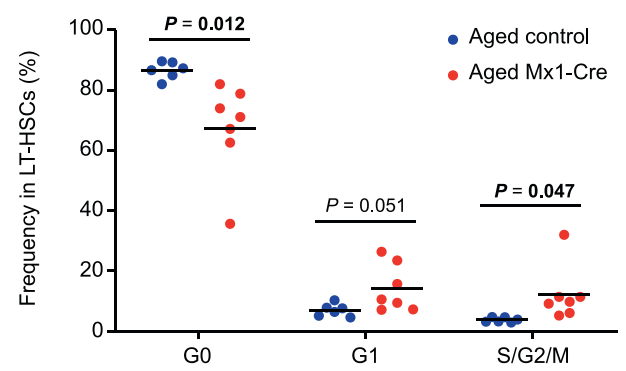

perifosine as well as rapamycin effectively decreased the mitochondrial membrane potential and intracellular ROS levels in HSPCs of young Vav-Cre ASXL1-MT KI mice (Supplementary Fig. 10a-f). We next assessed whether the enhanced Akt/mTOR signaling reduced engraftment of ASXL1-MT KI HSPCs. Bone
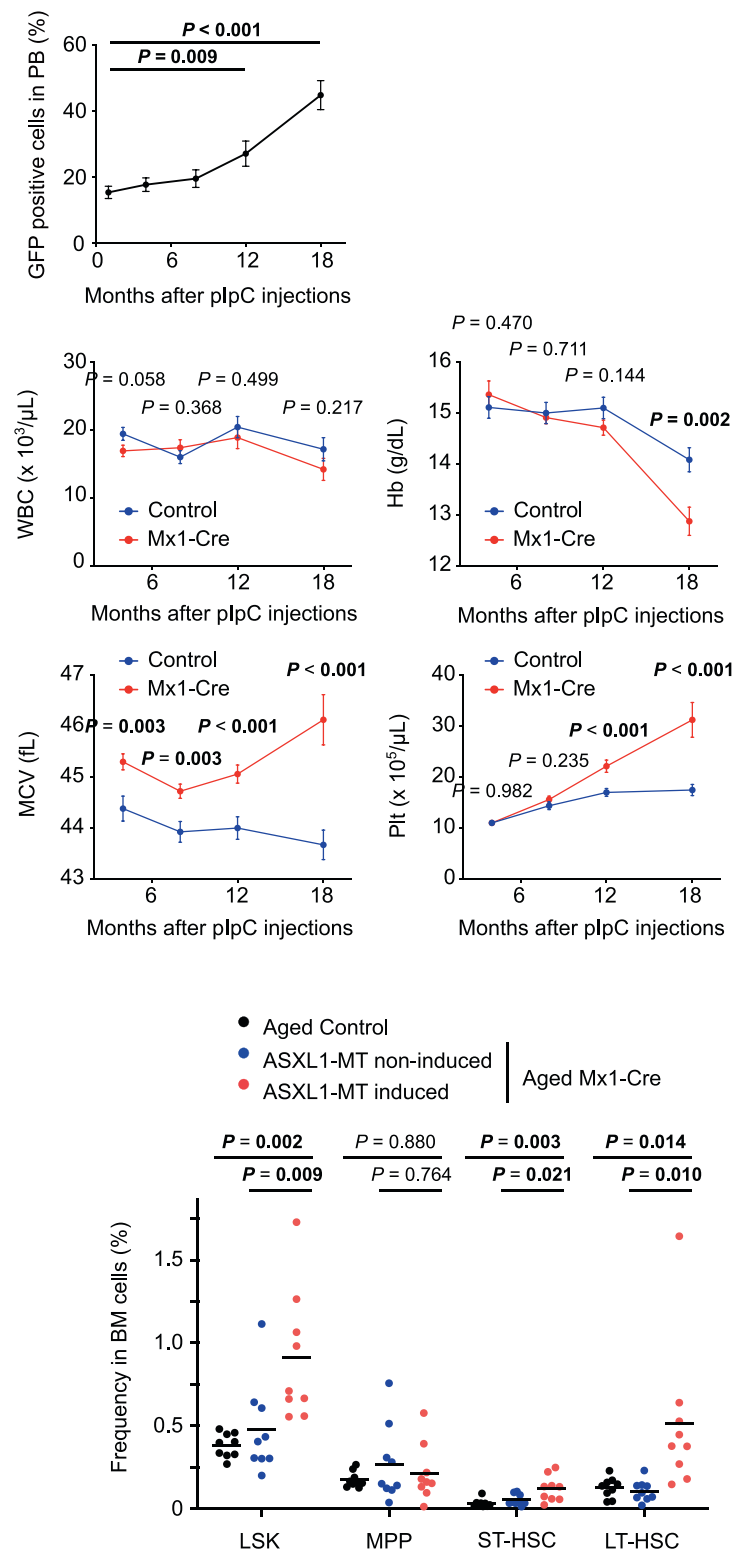

f

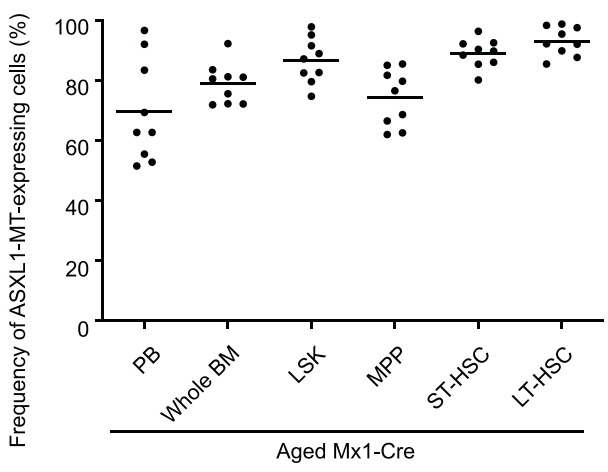

marrow cells from young Vav-Cre ASXL1-MT KI mice or littermate control mice treated with rapamycin were transplanted into recipient mice (Supplementary Fig. 10d). Rapamycin treatment effectively improved engraftment of ASXL1-MT-expressing bone marrow cells (Supplementary Fig. 10g). These data indicate 
Fig. 3 ASXL1-MT confers a fitness advantage on LT-HSCs with aging. a The experimental design for partial induction of ASXL1-MT in vivo using Mx1-Cre ASXL1-MT KI mice. Young Mx1-Cre ASXL1-MT KI mice were partially induced to express ASXL1-MT by plpC injections (10 $\mu \mathrm{g} / \mathrm{mouse} \times 5$ times) at 12 weeks after birth. 18 months after plpC injections, bone marrow cells from Mx1-Cre ASXL1-MT KI mice were analyzed. b The frequency of ASXL1-MT expressing cells in peripheral blood at the indicated months after plpC injections $(n=20)$. c Enumeration of white blood cells $(\mathrm{WBC})$, hemoglobin $(\mathrm{Hb}), \mathrm{mean}$ corpuscular volume (MCV), and platelets (PIt) in peripheral blood of Mx1-Cre ASXL1-MT KI mice at the indicated months after plpC injections ( $n=20$ ). d The frequency of LSK cells, MPPs, ST-HSCs and LT-HSCs in ASXL1-MT induced cells, ASXL1-MT non-induced cells, or whole bone marrow cells of agematched control mice $(n=9)$. Representative FACS plot (left panel) and summarized data (right panel) are shown. e Cell cycle analysis with Ki-67/DAPI staining of LT-HSCs of aged Mx1-Cre ASXL1-MT KI mice and age-matched control mice $(n=6)$. $\mathbf{f}$ The frequency of ASXL1-MT-expressing cells in peripheral blood, whole bone marrow, LSK, MPP, ST-HSC, and LT-HSC fractions of aged mice (18 months after plpC injections) $(n=9)$. Data are mean \pm s.e.m. Data are assessed by two-tailed Student's $t$-test $(\mathbf{b}, \mathbf{c}, \mathbf{e})$ or one-way ANOVA with Tukey-Kramer's post-hoc test $(\mathbf{d}) .{ }^{\star} P \leq 0.05,{ }^{\star \star} P \leq 0.01,{ }^{\star \star \star} P \leq 0.001$.

that enhanced Akt/mTOR-signaling induced by ASXL1-MT provokes mitochondrial activation, ROS overproduction, and dysfunction of HSPCs. It should be noted, however, that neither antioxidant nor rapamycin inhibits the myeloid-skewed hematopoiesis in young $\mathrm{Vav}$-Cre ASXL1-MT KI mice (Fig. 8e and Supplementary Fig. 10h). Therefore, it is likely that ASXL1-MT promotes myeloid skewing through Akt/mTOR-independent functions.

To examine the relationship between the enhanced Akt/mTOR signaling and dysregulated hematopoiesis in aged ASXL1-MT KI mice, we treated aged Vav-Cre ASXL1-MT KI mice and agematched control mice with rapamycin for 8 weeks (Fig. 9a). As was the case with young mice, mitochondrial membrane potential and intracellular ROS levels were increased in LT-HSCs of aged Vav-Cre ASXL1-MT KI mice compared with age-matched control mice (Fig. 9b and c). ASXL1-MT-induced elevation of mitochondrial membrane potential and ROS levels was further validated in LT-HSCs of aged Mx1-Cre ASXL1-MT KI mice (Supplementary Fig. 11a-c). Rapamycin treatment tended to reduce the mitochondrial membrane potential and normalized ROS levels in aged LT-HSCs expressing ASXL1-MT (Fig. 9b and c). Treatment with perifosine also abrogated mitochondrial activation in these cells (Supplementary Fig. 11d and e). Importantly, treatment with rapamycin recovered the number of differentiated white blood cells as well as bone marrow cellularity in aged Vav-Cre ASXL1-MT KI mice (Fig. 7d and e). Furthermore, mitochondrial activation, increased DNA damage, and differentiation defects in aged ASXL1-MT KI HSPCs were ameliorated by rapamycin treatment (Fig. 9f-j). Taken together, these data suggest that overactive Akt/mTOR pathway causes dysregulated hematopoiesis, whereas it confers a growth advantage on pLT-HSCs in aged ASXL1-MT KI mice.

ASXL1-MT enforces age-associated patterns of gene expression in LT-HSCs. To further characterize the impact of ASXL1-MT on physiological aging of LT-HSCs, we performed RNA-seq analysis using LT-HSCs from aged $\mathrm{Vav}$-Cre ASXL1-MT KI mice and agematched control mice (Fig. 10a and b). As was the case with young mice, GSEA suggests activation of Akt/mTOR pathway in aged Vav-Cre ASXL1-MT KI mice, in concordance with flow cytometry analyses (Figs. 4d, e, and 10c). In aged ASXL1-MTexpressing LT-HSCs, up-regulated genes significantly overlapped with genesets related to Akt/mTOR signaling, platelet signature, and senescence; conversely, down-regulated genes significantly overlapped with genesets related to myeloid differentiation, erythroid differentiation, and HSC signature (Fig. 10d). Since ASXL1-MT appears to accelerate aging of LT-HSCs (e.g. anemia, myeloid-skewed differentiation, and hypocellular bone marrow), we compared alteration of gene expression profiles associated with physiological aging of LT-HSCs (GSE48893) with this RNAseq analysis data. This analysis revealed that ASXL1-MT promotes the expression of age-associated patterns, suggesting that ASXL1-MT accelerates aging of LT-HSCs (Fig. 10e and f).
AKT/mTOR pathway seems to be activated in MDS patients harboring ASXL1 mutations compared with those harboring DNMT3A or TET2 mutations. Finally, we examined whether the development of MDS, a clonal HSC disease frequently arising from $\mathrm{CH}$, harboring ASXL1 mutations is associated with enhanced Akt/mTOR signaling. To this end, we analyzed a public transcriptome data of CD34-positive bone marrow cells from MDS patients with DNMT3A, TET2, or ASXL1 mutations, and healthy control subjects (Gene Expression Omnibus accession number: GSE58831). GSEA suggested that AKT pathway is upregulated in MDS patients harboring ASXL1 mutations, but not those harboring DNMT3A or TET2 mutations, compared to healthy control subjects (Supplementary Table 2). We also found downregulation of negatively regulated genes by mTOR signaling in MDS patients with ASXL1 mutations (Supplementary Table 2). These data suggest that AKT/mTOR pathway is activated in MDS patients harboring ASXL1 mutations.

\section{Discussion}

Somatic mutations in epigenetic regulators, ASXL1, TET2, and DNMT3A, are recurrently detected in $\mathrm{CH}^{19-21}$. TET2 or DNMT3A deficiency in mice enhances self-renewal of LT$\mathrm{HSCs}^{30-33}$, suggesting that mutations in TET2 and DNMT3A induce clonal expansion of hematopoietic cells, leading to $\mathrm{CH}$ in humans. In contrast, ASXL1 mutations in mice reduce the number and function of $\mathrm{HSCs}^{25,34,35}$. In the present study, we confirmed that ASXL1-MT causes a competitive disadvantage of LT-HSCs after transplantation. Nevertheless, LT-HSCs expressing ASXL1-MT acquire a growth advantage and eventually occupy the HSC compartment during aging in genetic mosaic mouse model. This observation is clearly distinct from that of DNMT3A or TET2 mutations, which exhibits an increase in the long-term regenerative potential of HSCs. Our results provide an insight into the pathogenesis of $\mathrm{CH}$, in that $\mathrm{CH}$ can be induced by clonal expansion of not only "true" HSCs but also by long-lived pLT-HSCs lacking repopulation potential ${ }^{1,9}$. The enhanced Akt/ mTOR pathway is expected to provoke replication stress along with increased ROS-mediated DNA damage. Indeed, we observed increased DNA strand breaks in aged ASXL1-MT KI pLT-HSCs, indicating that ASXL1-MT expands the pLT-HSC compartment with accumulation of DNA damage. This could be one mechanistic explanation for why individuals with $\mathrm{CH}$ are at increased risk for subsequent hematological malignancies, associated with secondary mutations.

The present results suggest that the activated Akt/mTOR pathway, which promotes cell cycle progression, is responsible for aberrant expansion of the LT-HSC compartment in aged ASXL1MT KI mice. At the same time, we also found that overactive Akt/ mTOR signaling induced by ASXL1-MT provokes dysfunction of HSCs. It has been shown that the enhanced Akt/mTOR activity by depletion of Pten results in transient expansion and subsequent depletion of $\mathrm{HSC}^{58,59}$. It appears that ASXL1-MT has a much milder impact on Akt/mTOR pathway than disruption of a 
a

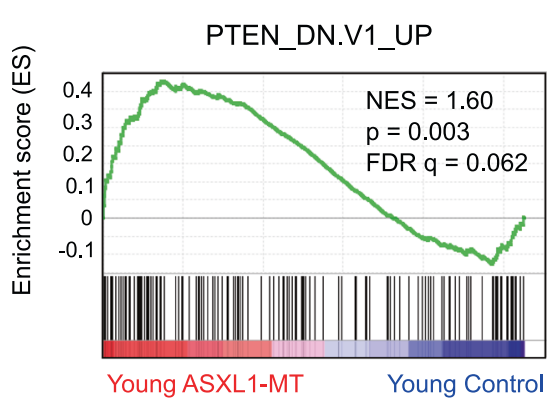

c

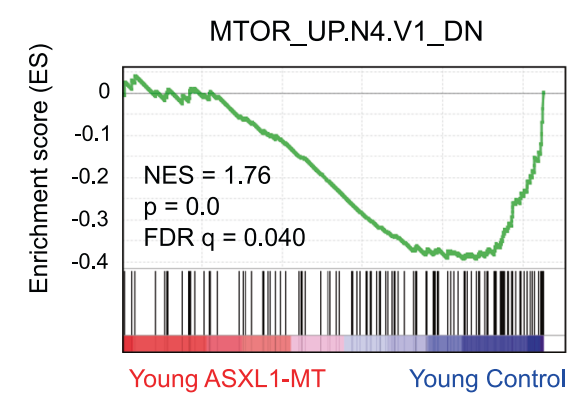

d

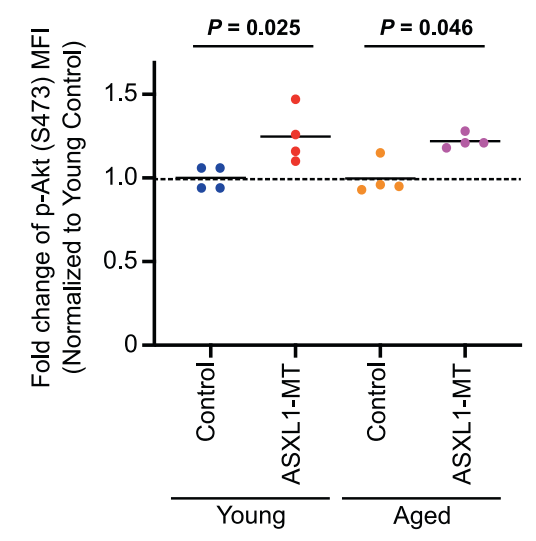

b

e
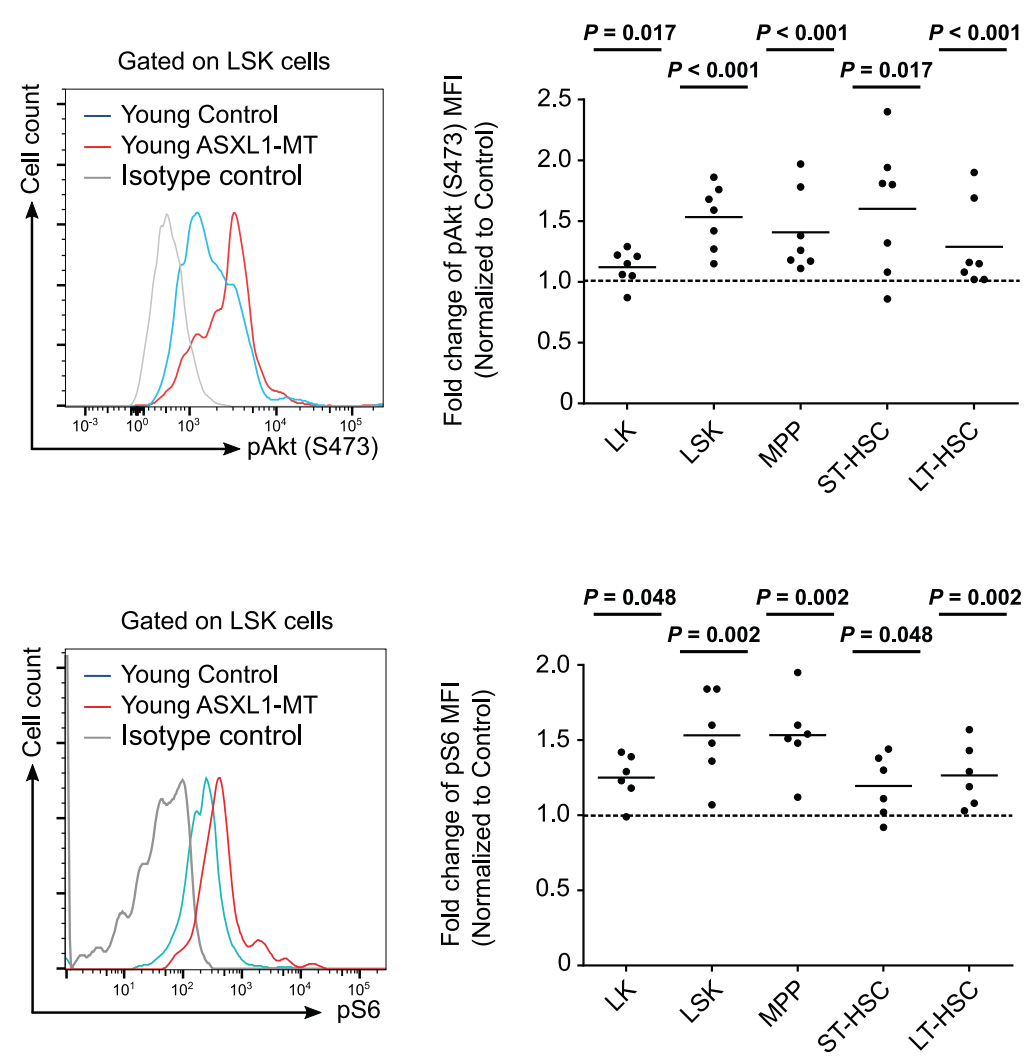

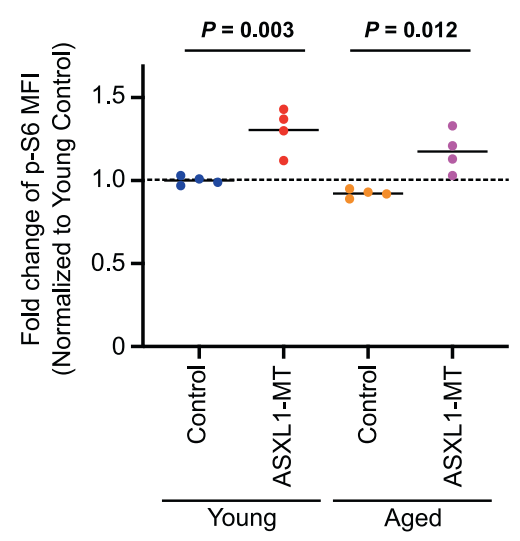

Fig. 4 ASXL1-MT activates Akt/mTOR pathway in both young and aged mice. a Gene set enrichment analysis of LSK cells from young Vav-Cre ASXL1MT KI mice and littermate control mice using genesets up-regulated by PTEN deficiency (PTEN_DN.V1_UP) and negatively regulated by mTOR signaling (MTOR_UP.N4.V1_DN) $(n=3)$. b, $\mathbf{c}$ Levels of phosphorylated Akt (S473) (b) and phorphorylated S6 (c) in LK cells, LSK cells, MPPs, ST-HSCs and LT-HSCs from young Vav-Cre ASXL1-MT KI mice $(n=7)$. Representative FACS plot (left panel) and summarized data (right panel) are shown. d, e Levels of phosphorylated Akt (S473) (d) and phosphorylated S6 (e) in LT-HSCs of young and aged Vav-Cre ASXL1-MT KI mice $(n=4)$. Statistical significances are assessed by two-tailed Mann-Whitney's $u$-test $(\mathbf{b}, \mathbf{c})$ or one-way ANOVA with Tukey-Kramer's post-hoc test $(\mathbf{d}, \mathbf{e}) .{ }^{\star} P \leq 0.05,{ }^{\star \star} P \leq 0.01,{ }^{\star \star \star} P \leq 0.001$.

core repressive component comprising Akt/mTOR pathway. The modestly activated AKT/mTOR signaling induced by ASXL1-MT presumably has negative effects on HSC function, whereas it causes abnormal expansion of pLT-HSCs in steady-state hematopoiesis over time. These molecular changes should underlie the expansion of pLT-HSCs along with impaired repopulation potential in ASXL1-MT KI mice. Related to these findings, we observed enhancement of age-associated phenotypes in hematopoiesis including anemia, myeloid-biased differentiation, hypocellular bone marrow, and expansion of surface marker-defined LT-HSCs in aged ASXL1-MT KI mice. These phenotypes were partly rescued by inhibition of Akt/mTOR pathway (Figs. $5 \mathrm{c}$ and 9d), suggesting that ASXL1-MT-induced activation of Akt/mTOR pathway is involved in enhanced aging of the hematopoietic system. In addition, RNA-seq analyses revealed that ASXL1-MT promotes the gene expression of age-associated patterns. These results indicate that ASXL1-MT could promote HSC aging via activation of Akt/mTOR pathway.

One of the most intriguing findings of this work is the identification of AKT as a non-histone target of BAP1. BAP1, which is stabilized and given an enhanced DUB activity by ASXL1-MT, is a known DUB for histone $\mathrm{H} 2 \mathrm{~A}$, and was also shown to deubiquitinate several non-histone proteins ${ }^{60-64}$. It was previously demonstrated that multiple E3 ligases induce ubiquitination and 


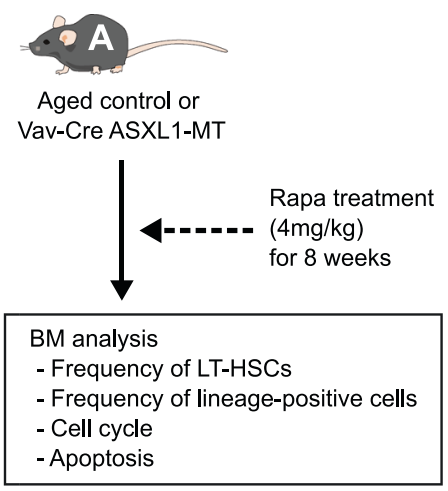

b

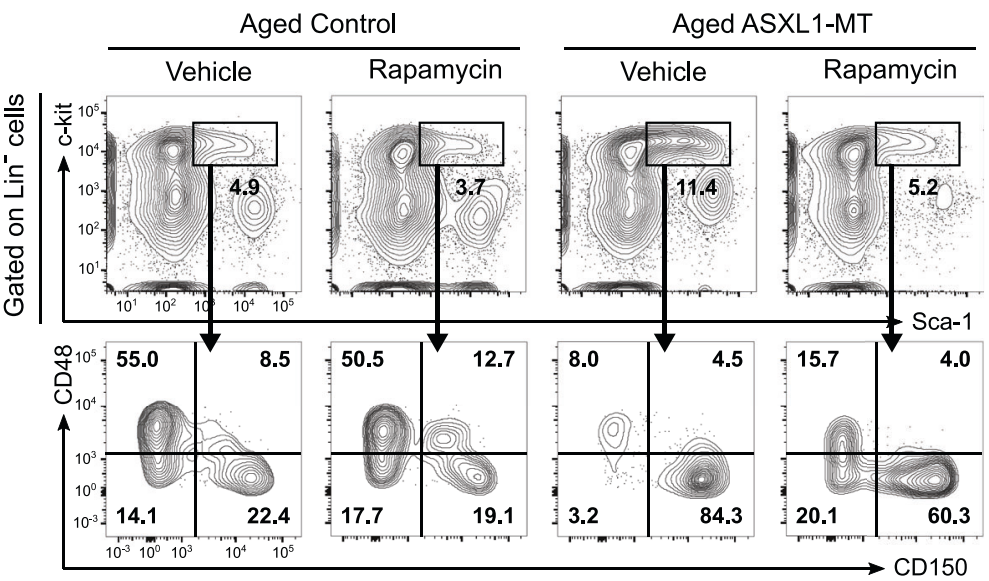

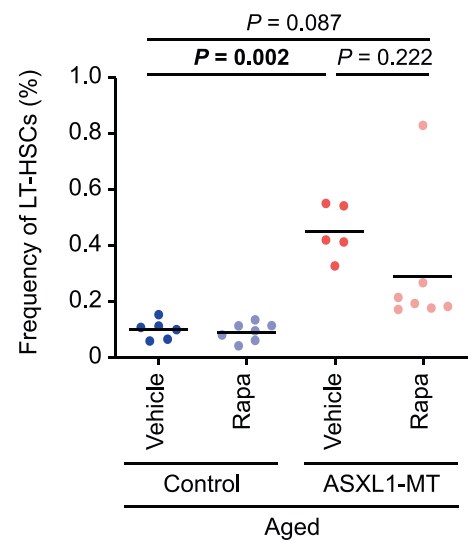

e

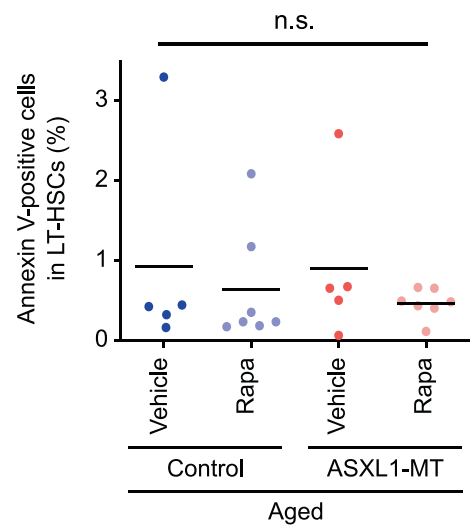

d

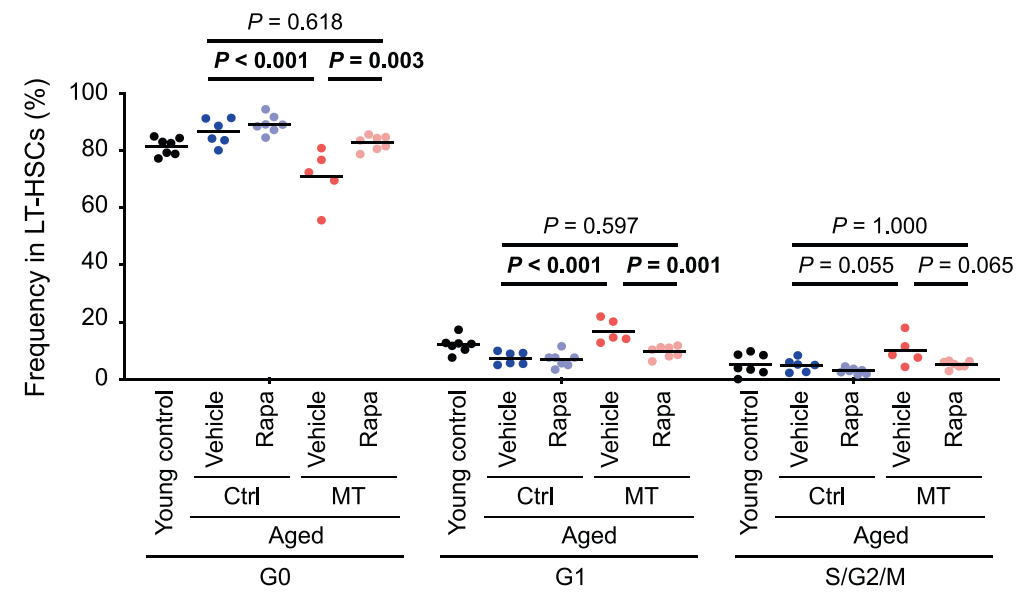

g

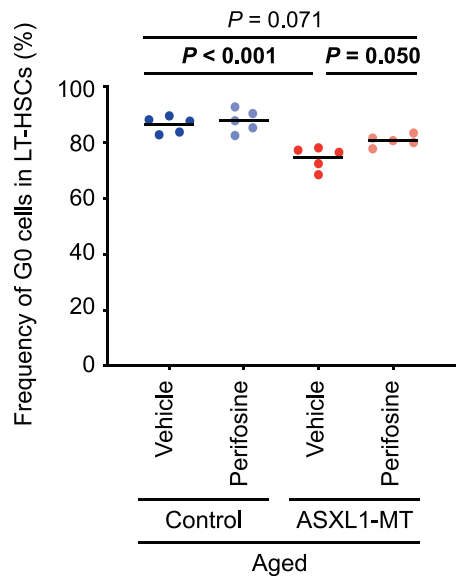

Fig. 5 Inhibition of Akt/mTOR pathway ameliorates ASXL1-MT-induced aberrant proliferation of pLT-HSCs. a The experimental design for treatment with an mTOR inhibitor rapamycin in aged mice. Aged Vav-Cre ASXL1-MT KI mice and age-matched control mice were treated with rapamycin intraperitoneally ( $4 \mathrm{mg} / \mathrm{kg} /$ day) every other day for 8 weeks. At the end of administrations, end-point analyses of bone marrow cells were conducted. b, $\mathbf{c}$ The frequency of LT-HSCs in bone marrow MNCs ( $n=6$ (Control-Vehicle), 7 (Control-Rapamycin), 5 (ASXL1-MT-Vehicle), and 7 (ASXL1-MTRapamycin)). Representative FACS plot (b) and summarized data (c) are shown. $\mathbf{d}$ Cell cycle analysis with Ki-67/DAPI staining of LT-HSCs ( $n=7$ (Young control), 6 (Control-Vehicle), 7 (Control-Rapamycin), 5 (ASXL1-MT-Vehicle), and 7 (ASXL1-MT-Rapamycin)). e Apoptosis analyses of LT-HSCs ( $n=5$ (Control-Vehicle), 7 (Control-Rapamycin), 5 (ASXL1-MT-Vehicle), and 7 (ASXL1-MT-Rapamycin)). f The experimental design for treatment with an Akt inhibitor perifosine in aged mice. Aged Vav-Cre ASXL1-MT KI mice and age-matched control mice were treated with a single oral dose (36 mg/kg) of perifosine $(n=5) . \mathbf{g} 16 \mathrm{~h}$ after administration, cell cycle analyses with Ki-67/DAPI staining of LT-HSCs were performed. Statistical significances are assessed by one-way ANOVA with Tukey-Kramer's post-hoc test. ${ }^{\star} P \leq 0.05,{ }^{\star \star} P \leq 0.01,{ }^{\star \star \star} P \leq 0.001$. 
a

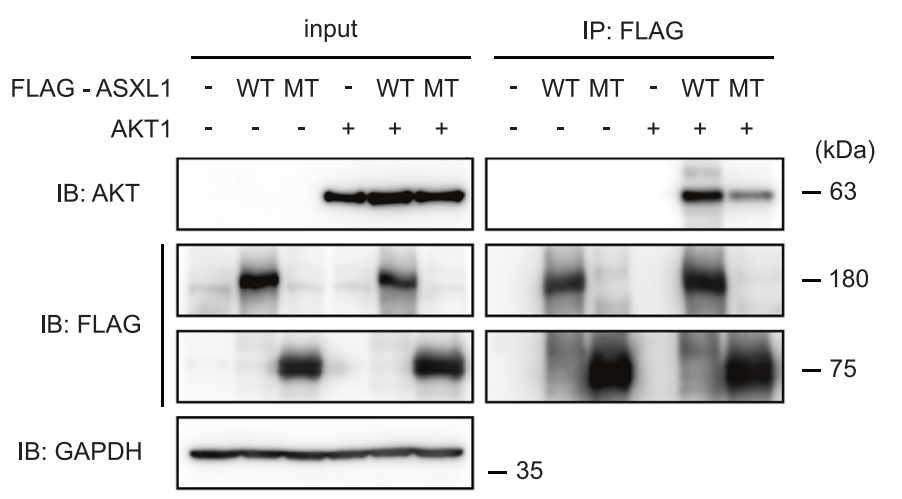

b

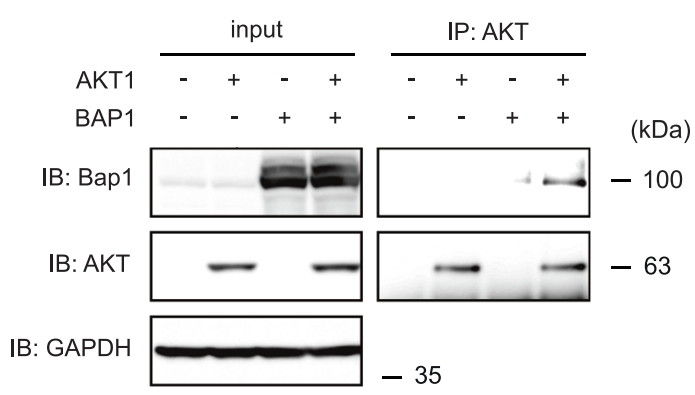

c

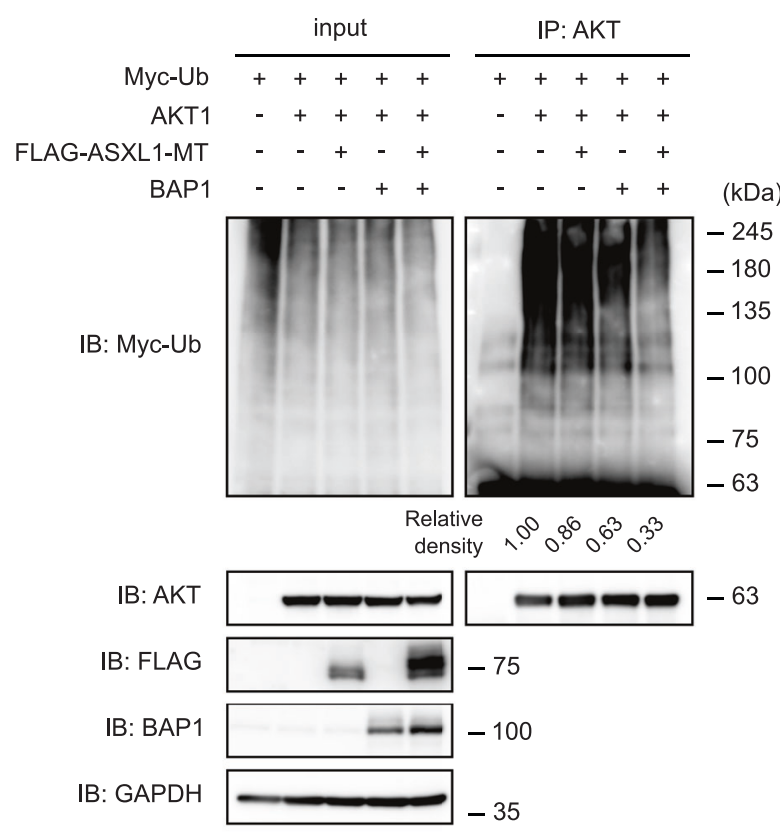

e

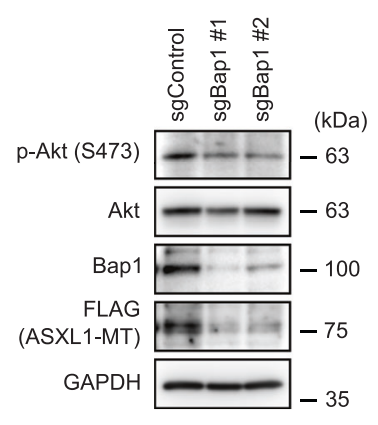

d

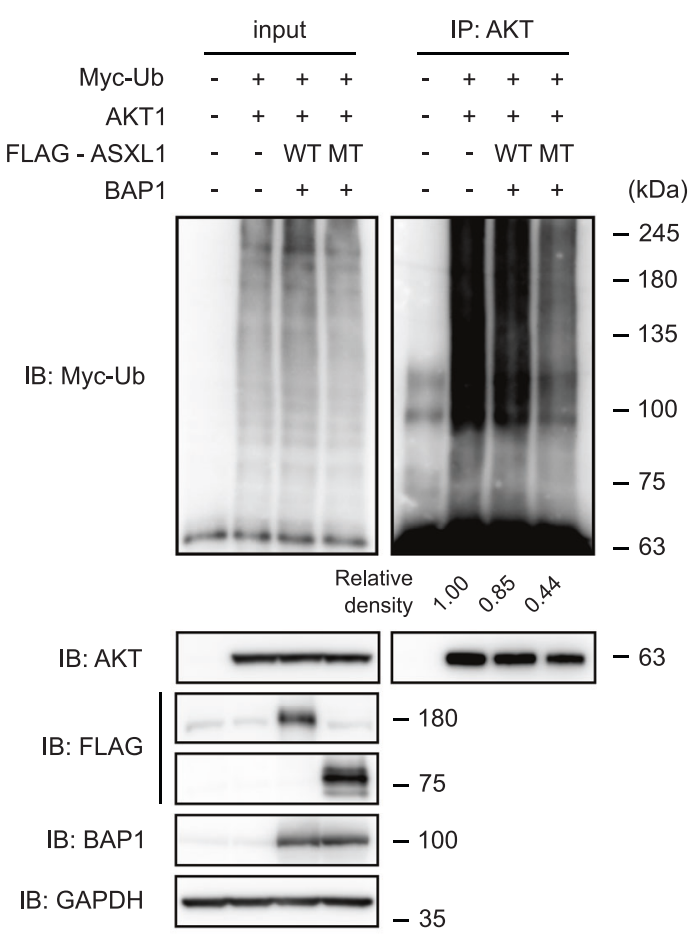

g

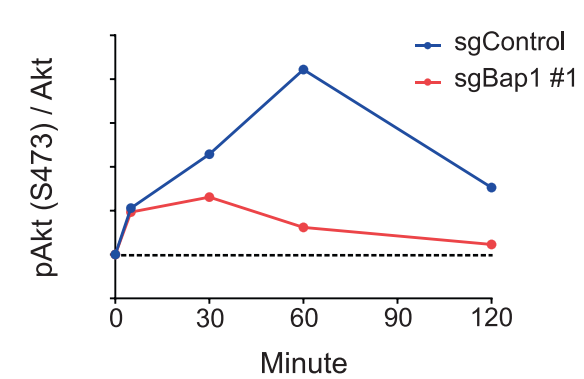

degradation of phosphorylated AKT, thereby quenching activated AKT signaling ${ }^{38,40-42}$. Conversely, ASXL1-MT/BAP1 complex deubiquitinates and stabilizes AKT, which leads to weak but possibly continuous activation of Akt/mTOR pathway. Thus, these findings revealed a function of ASXL1-MT as a signaling modulator beyond its known roles as an epigenetic regulator. On the other hand, one recent study has shown that Asxl1 loss activates Akt/ mTOR pathway due to an epigenetic dysregulation at the Pten locus ${ }^{65}$. Thus, we cannot completely exclude the possibility that
ASXL1-MT activates Akt/mTOR pathway through an epigeneticdependent manner as well.

Among individuals with $\mathrm{CH}$, the increased risk for developing hematological malignancies and cardiovascular diseases is associated with higher variant allele frequency ${ }^{21,66}$. Thus, it is possible to avoid the onset of these diseases by inhibiting the expansion of mutated $\mathrm{CH}$ clone. In the present study, we demonstrate that the aberrant cell cycle progression and the increased frequency of pLT-HSCs are suppressed by treatment with rapamycin in aged 
Fig. 6 ASXL1-MT/BAP1 complex deubiquitinates and stabilizes Akt. a 293T cells were transfected with FLAG-ASXL1-WT, FLAG-ASXL1-MT, and AKT1 expressing vectors. Total cell lysates were subjected to immunoprecipitation using anti-FLAG antibody followed by immunoblotting. $\mathbf{b} 293 \mathrm{~T}$ cells were transfected with AKT1 and BAP1 expressing vectors. Total cell lysates were subjected to immunoprecipitation using anti-AKT antibody followed by immunoblotting. c 293T cells were transfected with AKT1, FLAG-ASXL1-MT, BAP1, and Myc-Ubiquitin expressing vectors. Total cell lysates were subjected to immunoprecipitation using anti-AKT antibody followed by immunoblotting. Relative levels of Myc-Ubiquitin were quantified by densitometry and normalized to total Myc-Ubiquitin levels. d 293T cells were transfected with AKT1, FLAG-ASXL1-WT, FLAG-ASXL1-MT, BAP1, and Myc-Ubiquitin expressing vectors. Total cell lysates were subjected to immunoprecipitation using anti-AKT antibody followed by immunoblotting. Relative levels of MycUbiquitin were quantified by densitometry and normalized to total Myc-Ubiquitin levels. e-g Murine bone marrow cells transformed by combined expression of SETBP1-D868N and ASXL1-MT (cSAM cells) were transduced with Cas9 and sgRNA targeting Bap1 (e). Bap1-depleted cSAM cells were then starved of IL-3 for $3 \mathrm{~h}$ followed by stimulation with IL-3 $(1 \mathrm{ng} / \mathrm{mL})$ for the indicated times (f). Relative levels of phosphorylated Akt were quantified by densitometry and normalized to total Akt levels (g). Similar result was obtained from Bap1-depleted cSAM cells using another sgRNA-targeting BAP1 (sgBAP1 \#2). A representative experiment from at least $n=2$ independent experiments is shown.

ASXL1-MT KI mice. Hence, a pharmacological inhibition of the Akt/mTOR pathway to individuals with $\mathrm{CH}$ harboring ASXL1 mutations is expected to reduce a clonal advantage of the $\mathrm{CH}$ clone.

Besides $\mathrm{CH}$, recent reports have revealed that normal tissues also accumulate somatic mutations with aging, which may confer a selective advantage 67 . However, mutational landscapes are clearly distinct; while mutations in epigenetic factors and splicing factors are frequently detected in $\mathrm{CH}$, most mutations detected in oncogenes are signal transduction molecules in other tissues. At present, the reason for this discrepancy remains totally elusive. Clarification of the mechanisms by which $\mathrm{CH}$ progress to hematological malignancies may provide us a clue to understand the difference.

In conclusion, we show that ASXL1-MT collaborates with BAP1 to activate Akt/mTOR pathway in an epigeneticsindependent manner. The activated Akt/mTOR pathway causes aberrant expansion of pLT-HSCs to occupy the HSC compartment during aging. Overactive Akt/mTOR signaling also causes mitochondrial activation, overproduction of ROS, increased DNA damage, and subsequent dysfunction of HSCs. The expansion of pLT-HSCs along with increased DNA damage caused by ASXL1 mutations can result in the development of $\mathrm{CH}$, leading to hematopoietic malignancy with secondary mutations. A pharmacological inhibition of Akt/mTOR pathway may pave the way for a preventive intervention to individuals with $\mathrm{CH}$ harboring ASXL1 mutations.

\section{Methods}

Mice. Wild-type C57BL/6J mice were bred in-house. $p 53^{-1-}$ mice were provided by K. Matsuda (Tokyo University). Conditional ASXL1-MT KI mice have been generated in our laboratory and crossed to Vav-Cre or Mx1-Cre transgenic mice. Experiments were performed with 6-12-week-old for young mice or 20-24-monthold for aged mice. All mice were housed with a $12 \mathrm{~h}$ dark/light cycle at a temperature between $20-25^{\circ} \mathrm{C}$ and a humidity between $40 \%$ and $60 \%$. The experiments were approved by the Committee on the Ethics of Animal Experiments and all these mice were maintained according to the guidelines of the Institute of Laboratory Animal Science (PA13-19 and PA16-31).

$\mathbf{N}$-acetyl-L-cysteine treatment. To examine the effect of ROS on repopulation ability of HSPCs, ASXL1-MT KI mice and littermate control mice were treated with drinking water containing $1 \mathrm{mg} / \mathrm{mL}$ N-acetyl-L-cysteine (NAC) (SIGMA, A7250) for 8 weeks before transplantation. Recipient mice were continuously treated with NAC after transplantation.

Rapamycin treatment. Rapamycin (LC Laboratories, R-5000) was dissolved in DMSO (20 mg/mL) and further diluted with 10\% PEG-300 (SIGMA, 202371)/5\% Tween 80 (SIGMA, P4780)/PBS. Mice were treated with $4 \mathrm{mg} / \mathrm{kg}$ rapamycin intraperitoneally as described in figure legends.

Perifosine treatment. Perifosine (Chemscene LLC, CS-0209) was dissolved in sterile water, and $36 \mathrm{mg} / \mathrm{kg}$ was administered orally $16 \mathrm{~h}$ before analyses.

polyinosine-polycytidine (plpC) treatment. Mx1-Cre ASXL1-MT KI mice were intraperitoneally injected with $250 \mu \mathrm{g}$ pIpC (SIGMA, P1530) three times every other day for competitive transplantations, or $10 \mu \mathrm{g}$ pIpC 5 times every other day for partial induction of ASXL1-MT at 12 weeks after birth.

Plasmids. The SF91-IRES-GFP and SF91-Cat/SOD2-IRES-GFP vectors were kindly provided by Michael D. Milsom. SF91-IRES-Venus and SF91-Cat/SOD2IRES-Venus vectors were generated by replacing GFP with Venus sequence at the PmlI/BsrGI sites. The 3xFLAG-tagged ASXL1-WT and ASXL1-MT (1900-1922del E635RfsX15) were cloned into pMYs-IRES-GFP vector as previously described ${ }^{23}$. The HA-tagged BAP1 was cloned into pMYs-IRES-NGFR vector as previously described ${ }^{23}$. T7-p53DD-pcDNA3 (Addgene \#25989) was obtained from Addgene, and we cloned it to pMYs-IRES-NGFR vector as previously described ${ }^{68}$. The HAtagged AKT1 was cloned into pMXs-IRES-GFP vector at the BamHI/EcoRI sites.

Cell culture. 293T cells (CRL-11268, ATCC, Manassas, VA, USA) were cultured in Dulbecco's modified Eagle's medium (DMEM) supplemented with $10 \%$ fetal bovine serum (FBS) and 1\% penicillin/streptomycin. cSAM cells were generated by transducing SETBP1-D868N and ASXL1-MT into murine bone marrow progenitors and were cultured in RPMI1640 medium supplemented with $10 \%$ FBS, IL-3 ( $1 \mathrm{ng} / \mathrm{mL})$, and $1 \%$ penicillin/streptomycin ${ }^{45}$.

Retroviral bone marrow transplantation. Plat-E packaging cells were transfected with retroviral constructs using calcium phosphate co-precipitation method. After transfection, retroviral supernatant was collected and loaded onto RetroNectincoated plates. Freshly isolated Lin $^{-}$cells were cultured in Iscove's modified Dulbecco's media (IMDM) supplemented with $20 \%$ FBS, 2 mM L-glutamine, SCF (50 $\mathrm{ng} / \mathrm{mL})$, Flt3-ligand $(50 \mathrm{ng} / \mathrm{mL})$, and TPO $(50 \mathrm{ng} / \mathrm{mL})$ overnight, and were subsequently transduced with the viruses for $48 \mathrm{~h}$.

CRISPR/Cas9-mediated gene knockout. To obtain short guide RNA (sgRNA) constructs targeting for Bap1, annealed oligos were cloned into lentiGuide-puro vector (Addgene, \#52963) as previously described ${ }^{23}$. 293T cells were transfected with Cas9-expressing vector (lentiCas9-Blast; Addgene, \#52962) or sgRNAexpressing vector together with lentiviral packaging vectors (pMD2.G; Addgene, \#12259 and psPAX2; Addgene, \#12260) using polyethylenimine (PEI), and then lentiviral supernatant was collected. cSAM cells were transduced with the viruses for $24 \mathrm{~h}$ and were subsequently subjected to drug selection with $10 \mu \mathrm{g} / \mathrm{mL}$ blasticidin for stable expression of Cas 9 and $1.5 \mu \mathrm{g} / \mathrm{mL}$ puromycin for stable expression of sgRNA.

Flow cytometry. Bone marrow cells were obtained by flushing long bones (femurs and tibias) in phosphate-buffered saline (PBS) containing $2 \%$ heat-inactivated FBS (FACS buffer). Cell suspensions were lysed with erythrocyte lysis buffer (150 mM $\mathrm{NH}_{4} \mathrm{Cl}, 10 \mathrm{mM} \mathrm{KHCO} 3,100 \mu \mathrm{M}$ EDTA-Na 2 ), filtered through a $40 \mu \mathrm{m}$ filter, and were incubated with a cocktail of biotinylated monoclonal antibodies to lineage markers (CD5, B220, CD11b, Gr-1, and Ter119) and anti-biotin microbeads (Miltenyi Biotec). Lin ${ }^{-}$cells were purified using MACS separation LS columns (Miltenyi Biotec). Cells were then stained with CD150-PE (BioLegend, 115904), ckit-PE-Cy7 (BioLegend, 105814), Sca1-APC (BioLegend, 108112), CD48-Brilliant Violet 421 (BioLegend, 103427), and Streptavidin-Brilliant Violet 605 (BioLegend, 405229). Expression levels of CD41 were analyzed using CD41-APC (BioLegend, 133914) antibody. Peripheral blood was analyzed using CD11b-PE (eBiosciense, 12-0112-85), CD45R/B220-PE/Cy7 (BioLegend, 103224), CD3-APC (100236), and CD45.2-APC/Cy7 (BioLegend, 109824) antibodies. For ROS, mitochondrial membrane potential and intracellular staining analyses, Scal-Brilliant Violet 785 (BioLegend, 108139) antibody was used instead of Sca1-APC antibody. For apoptosis, cell cycle and CD41 analyses, CD48-APC-Cy7 (BioLegend, 103431) and Sca1-Brilliant Violet 785 antibodies were used instead of CD48-Brilliant Violet 421 and Sca1-APC antibodies. Propidium iodide (PI) or 4',6-diamidino-2-phenylindole (DAPI) was used to exclude dead cells. All data were collected using a FACSVerse 

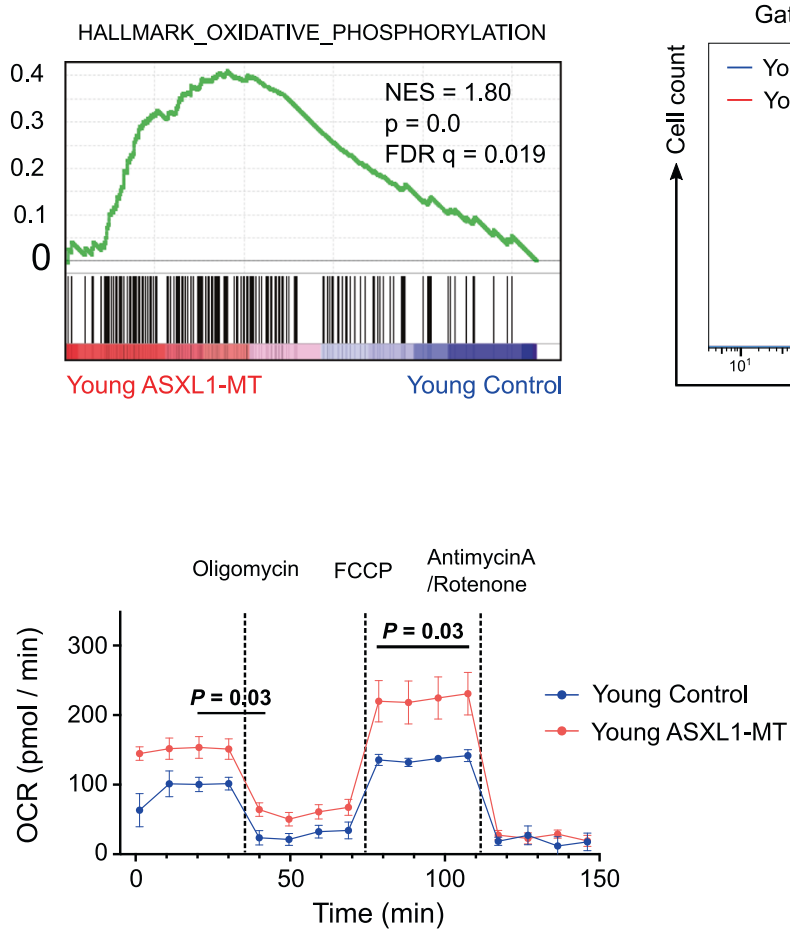

- Young Control - Young ASXL1-MT
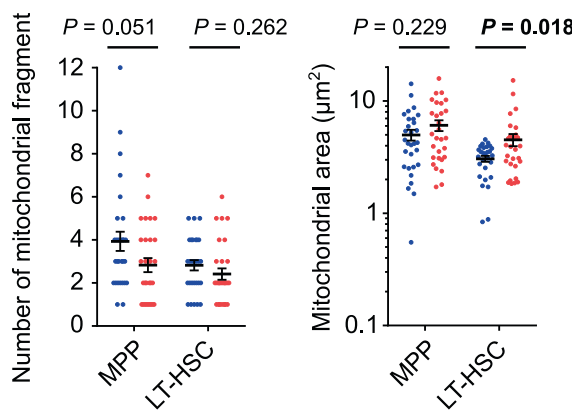

$\mathbf{f}$

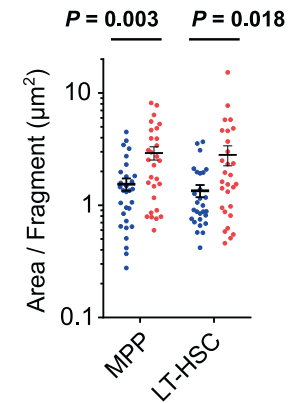

g
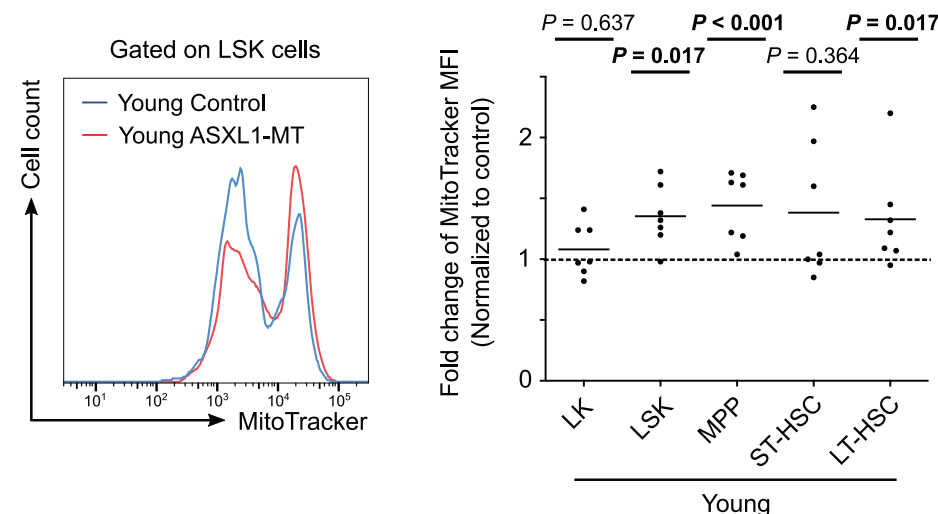

d

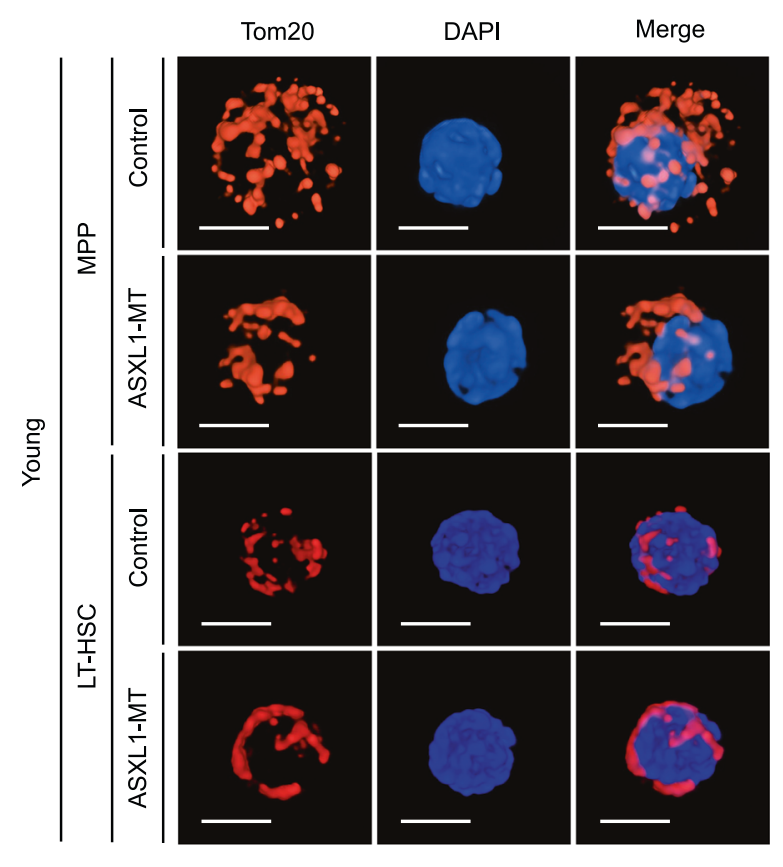

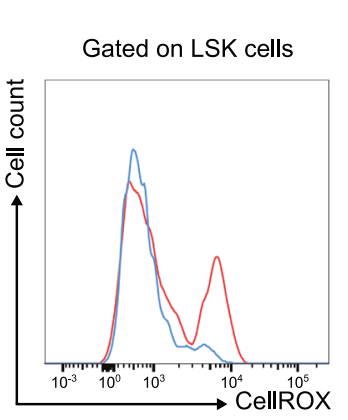
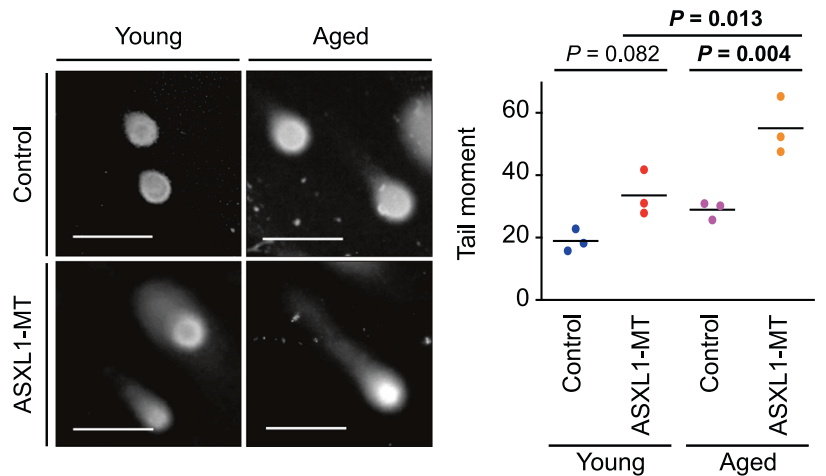

with BD FACSuite software or FACSAria with FACSDiva software and were analyzed with FlowJo software.

Intracellular staining. Bone marrow cells stained for surface markers were fixed in $4 \%$ paraformaldehyde for $10 \mathrm{~min}$ at room temperature, permeabilized with $0.2 \%$ Triton X-100 for $15 \mathrm{~min}$ at room temperature and blocked with $5 \%$ goat serum for
$15 \mathrm{~min}$ at room temperature. Cells were then incubated with anti-p-H2A.X (Cell Signaling Technology, \#9718, 1:200), anti-Akt (Pan) (Cell Signaling Technology, \#4691, 1:400), anti-Phospho-Akt (Ser473) (Cell signaling Technology, \#4060, 1:400), anti-pS6 (Ser235/236) (Cell Signaling Technology, \#4858, 1:100), or isotype control (abcam, ab172730) antibody for $1 \mathrm{~h}$ at room temperature, respectively. Cells were then washed and incubated with Alexa Fluor 647 goat anti-rabbit IgG (Invitrogen, A21245, 1:2000) for $1 \mathrm{~h}$ at room temperature. 
Fig. 7 ASXL1-MT induces mitochondrial activation, overproduction of ROS, and increased DNA damage. a Gene set enrichment analysis of TCA cycle and respiratory electron transport gene sets in LSK cells of young control and Vav-Cre ASXL1-MT KI mice $(n=3)$. b Mitochondrial membrane potential in LK cells, LSK cells, MPPs, ST-HSCs and LT-HSCs of young control and Vav-Cre ASXL1-MT KI mice $(n=7)$. Representative histogram in LSK cells is shown (left panel). Summarized results are expressed as fold change relative to their respective counterparts in littermate controls (right panel). c Oxygen consumption rates (OCR) in pooled $3 \times 10^{5} \mathrm{Lin}^{-} \mathrm{c}-\mathrm{kit}^{+}$cells under basal conditions and in response to oligomycin, fluoro-carbonyl cyanide phenylhydrazone (FCCP) and antimycinA/rotenone. Cells were obtained from three animals per genotype. Data are mean \pm s.e.m. d Representative immunofluorescence for Tom20 in MPPs and LT-HSCs (scale bars, $5 \mu \mathrm{m}$ ). e Quantification of mitochondrial fragment (left panel), area (middle panel), and area normalized to one fragment (right panel) (30 cells/group). Cells were obtained from 2 animals per genotype. Data are mean \pm s.e.m. $\mathbf{f}$ Intracellular ROS levels in LK cells, LSK cells, MPPs, ST-HSCs, and LT-HSCs of young control and Vav-Cre ASXL1-MT KI mice $(n=7)$. Representative histogram in LSK cells is shown (left panel). Summarized results are expressed as fold change relative to their respective counterparts in littermate controls (right panel). g Comet analyses using LT-HSCs from young and aged Vav-Cre ASXL1-MT KI mice $(n=3)$. Representative images (scale bars, $20 \mu \mathrm{m})$ (left panel) and olive tall moment (right panel) are shown. Statistical significances are assessed by two-tailed Mann-Whitney's $u$-test (b, $\mathbf{f})$, two-tailed Student's $t$-test $(\mathbf{c}, \mathbf{s} \mathbf{e})$, or one-way ANOVA with Tukey-Kramer's post-hoc test $(\mathbf{g}) .{ }^{\star} P \leq 0.05,{ }^{\star \star} P \leq 0.01$.

Cell cycle analysis. Bone marrow cells stained for surface markers were fixed in $4 \%$ paraformaldehyde for $10 \mathrm{~min}$ at room temperature, permeabilized with $0.2 \%$ Triton X-100 for $15 \mathrm{~min}$ at room temperature and blocked with $5 \%$ goat serum for $15 \mathrm{~min}$ at room temperature. Cells were then stained with the Ki-67-eFluor660 (eBiosciense, 50-5698-82) and DAPI for $30 \mathrm{~min}$ at $4^{\circ} \mathrm{C}$.

Apoptosis analysis. Bone marrow cells were labeled with surface marker antibodies, as described previously, and were then stained with Annexin V-APC (BioLegend, 640920) antibody in binding buffer (10 mM Hepes (pH 7.4), $140 \mathrm{mM}$ $\mathrm{NaCl}, 2.5 \mathrm{mM} \mathrm{CaCl}_{2}$ ) for $30 \mathrm{~min}$ at room temperature.

HSPC transplantation assay. Lethally irradiated ( $900 \mathrm{cGy}$ total, divided into two doses of $450 \mathrm{cGy}$ given over $4 \mathrm{~h}$ ) C57BL/6 recipient mice (CD45.1) were transplanted with either 200 LT-HSCs or 3000 MPPs (CD45.2) in competition with $4.0 \times 10^{5} \mathrm{C} 57 \mathrm{BL} / 6$ bone marrow mononuclear cells (MNCs) (CD45.1).

Serial transplantation assay. $1.0 \times 10^{6}$ bone marrow MNCs harvested from ASXL1-MT KI mouse were mixed with $1.0 \times 10^{6}$ bone marrow MNCs harvested from littermate control mouse and injected intravenously into lethally irradiated C57BL/ 6 recipient mice. 6 months after transplantation, $6 \times 10^{6}$ million bone marrow MNCs were isolated from three recipient mice and serially transplanted into lethally irradiated recipients. Donor-derived cells were evaluated by GFP positivity.

Homing assay. $5 \times 10^{4}$ LSK cells harvested from Vav-Cre ASXL1-MT KI mice were stained with $1 \mu \mathrm{M}$ CellTrace Far Red (Thermo Fisher Scientific, C34564) for $20 \mathrm{~min}$ at room temperature. Cells were then incubated with IMDM, washed, and injected into lethally irradiated mice. $16 \mathrm{~h}$ after transplantation, bone marrow cells isolated from femurs and tibias of recipient mice were analyzed to count CellTracepositive cells.

Quantification of cytokines. The levels of TNF- $\alpha$ and IFN- $\gamma$ in blood serum and bone marrow fluids were measured with TNF-alpha Quantikine ELISA Kit (R\&D Systems, MTA00B) and IFN-gamma Quantikine ELISA Kit (R\&D Systems, MIF00), respectively, according to the manufacturer's protocol.

Analysis of ROS levels. For intracellular ROS analysis, bone marrow cells were incubated for $30 \mathrm{~min}$ at $37^{\circ} \mathrm{C}$ with $5 \mu \mathrm{M}$ CellROX Deep Red (Thermo Fisher Scientific). Cells were then washed and analyzed by flow cytometry.

Quantification of mitochondrial membrane potential. Bone marrow cells were incubated with $100 \mu \mathrm{M}$ MitoTracker Red (Thermo Fisher Scientific) for $15 \mathrm{~min}$ at $37^{\circ} \mathrm{C}$. Cells were then washed and analyzed by flow cytometry.

Measurement of OCR. Sort-purified $3 \times 10^{5} \mathrm{Lin}^{-} \mathrm{c}$-kit ${ }^{+}$cells were immobilized to XF24 cell culture microplates pretreated with CELL-TAK (CORNING). Mitochondrial respiration was measured with a XF24 extracellular flux analyzer and XF cell Mito Stress Test Kit (Agilent). OCR was measured under basal conditions, followed by sequential injection of the ATP synthase inhibitor oligomycin $(0.5$ $\mu \mathrm{M}$ ), the uncoupler FCCP (trifluorocarbonylcyanide phenylhydrazone; $1.0 \mu \mathrm{M}$ ), the mitochondrial complex III inhibitor antimycin $\mathrm{A}(0.5 \mu \mathrm{M})$ and mitochondrial complex I inhibitor rotenone $(0.5 \mu \mathrm{M})$.

Immunofluorescence. MPPs or LT-HSCs were sorted into the slide coated with poly-L-lysine (SIGMA). Cells were placed on ice for $30 \mathrm{~min}$ to permit cells to settle onto the slide and fixed with $4 \%$ paraformaldehyde for $20 \mathrm{~min}$ at room temperature. Cells were then permeabilized with $0.2 \%$ TritonX-100 for $15 \mathrm{~min}$ at room temperature and blocked with $5 \%$ goat serum for $1 \mathrm{~h}$ at room temperature. Cells were incubated with the anti-p-H2A.X (Cell Signaling Technology, \#9718, 1:200) or anti-Tom20 (Santa Cruz Biotechnology, sc-11415, 1:500) antibody overnight at $4{ }^{\circ} \mathrm{C}$, washed and incubated in the Alexa Fluor 568 goat anti-rabbit IgG (Invitrogen, A11011, 1:1000) for $1 \mathrm{~h}$ at room temperature. Cell nuclei were stained with DAPI and mounted using fluorescence mounting medium (DAKO). Images were acquired with a Confocal Microscope A1R or super resolution microscope N-SIM (Nikon), and were processed with NIS-Elements software (Nikon). For quantitative analysis of mitochondrial morphology, images were first thresholded and then converted to binary images by using ImageJ software ${ }^{69,70}$. Individual particle (mitochondria) were analyzed for mitochondrial area and number of fragments.

Metabolome analysis. Sort-purified $5 \times 10^{4}$ LSK cells from three mice were pooled, washed and then frozen. Metabolite extraction from sorted cells for metabolome analysis was performed as described previously ${ }^{71}$. Briefly, frozen sorted cell fractions together with internal standard (IS) compounds (2-morpholinoethanesulfonic acid (MES) and 1,3,5-benzenetricarboxylic acid (trimesate)) was suspended in ice-cold methanol $(500 \mu \mathrm{L})$ followed by the addition of an equal volume of chloroform and 0.4 times the volume of ultrapure water (LC/MS grade, Wako). The suspension was then centrifuged at $15,000 \times g$ for $15 \mathrm{~min}$ at $4{ }^{\circ} \mathrm{C}$. After centrifugation, the aqueous phase was filtered using an ultrafiltration tube (Ultrafree MC-PLHCC, Human Metabolome Technologies). The filtrate was concentrated with a vacuum concentrator (SpeedVac, Thermo Fisher Scientific). The concentrated filtrate was dissolved in $25 \mu \mathrm{L}$ of ultrapure water and used for ion chromatography (IC)-MS analyses as described below. For metabolome analysis focused on TCA-cycle intermediates, anion metabolites were measured using an orbitrap-type MS (Q-Exactive focus, Thermo Fisher Scientific), connected to a high performance IC system (ICS-5000+, Thermo Fisher Scientific) that enables highly selective and sensitive metabolite quantification owing to the IC-separation and the Fourier Transfer MS principle ${ }^{72}$. The IC was equipped with an anion electrolytic suppressor (Thermo Scientific Dionex AERS 500, Thermo Fisher Scientific) to convert the potassium hydroxide gradient into pure water before the sample enters the mass spectrometer. The separation was performed using a Thermo Scientific Dionex IonPac AS11-HC, 4- $\mu \mathrm{m}$ particle size column. IC flow rate was $0.25 \mathrm{~mL} / \mathrm{min}$ supplemented post-column with $0.18 \mathrm{~mL} / \mathrm{min}$ makeup flow of $\mathrm{MeOH}$. The potassium hydroxide gradient conditions for IC separation are as follows: from 1 to $100 \mathrm{mM}(0-40 \mathrm{~min}), 100 \mathrm{mM}(40-50 \mathrm{~min})$, and $1 \mathrm{mM}(50.1-60 \mathrm{~min})$, at a column temperature of $30^{\circ} \mathrm{C}$. The $\mathrm{Q}$ Exactive focus mass spectrometer was operated under an ESI-negative mode for all detections. Full mass scan $(\mathrm{m} / \mathrm{z} 70-900)$ was used at a resolution of 70,000. The automatic gain control (AGC) target was set at $3 \times 10^{6}$ ions, and maximum ion injection time (IT) was $100 \mathrm{~ms}$. Source ionization parameters were optimized with the spray voltage at $3 \mathrm{kV}$ and other parameters were as follows: transfer temperature at $320^{\circ} \mathrm{C}$, S-Lens level at 50, heater temperature at $300^{\circ} \mathrm{C}$, Sheath gas at 36 , and Aux gas at 10 .

RNA-seq analysis. Total RNA was isolated from sorted LSK cells using RNeasy Micro-kit (QIAGEN) and Agilent TapeStation high sensitivity RNA ScreenTape or Bioanalyzer RNA 6000 Pico kit (Agilent) was used to check for RNA quality and quantity. RNA libraries for young mice were prepared using 15 ng total RNA with the QIAseq FX Single cell RNA library kit (QIAGEN) according to the manufacturer's protocol. In this protocol polyA-selected mRNA was converted in CDNA, and then enzymatic fragmentation (incubation time for "fragment size $=300 \mathrm{bp}$ " was used) and library preparation were performed using $1 \mu \mathrm{g}$ cDNA. RNA libraries for aged mice were prepared using $1 \mathrm{ng}$ total RNA with the NEBNext Single Cell/ Low Input RNA Library Prep kit (New England Biolabs). Briefly, cDNA synthesis and amplification were performed by template-switching PCR, and libraries were prepared using the amplified cDNA fragments. The quality and quantity of these libraries were checked using Agilent TapeStation D1000 or Agilent high sensitivity DNA kit (Agilent) and KAPA Library Quantification Kits (KAPA BioSystems)/ real-time PCR Systems Step One Plus (Applied Biosystems). These libraries were 


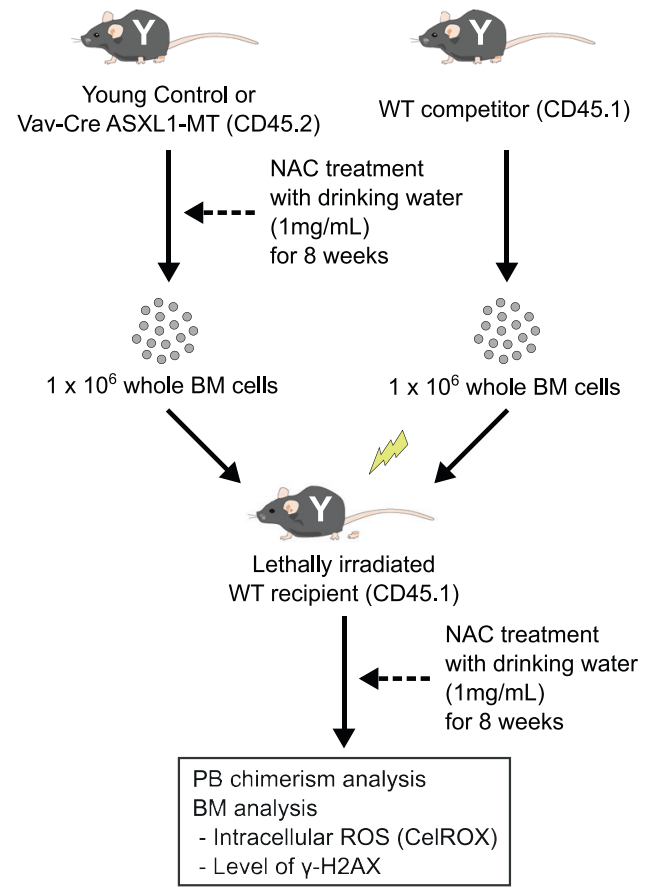

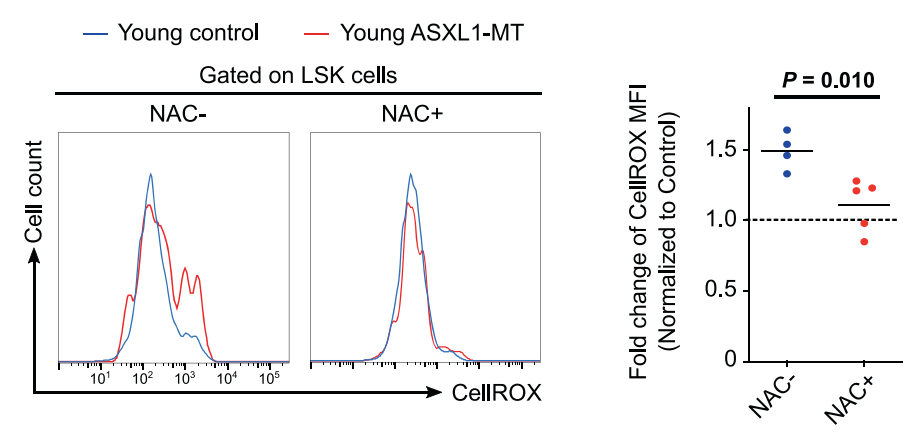
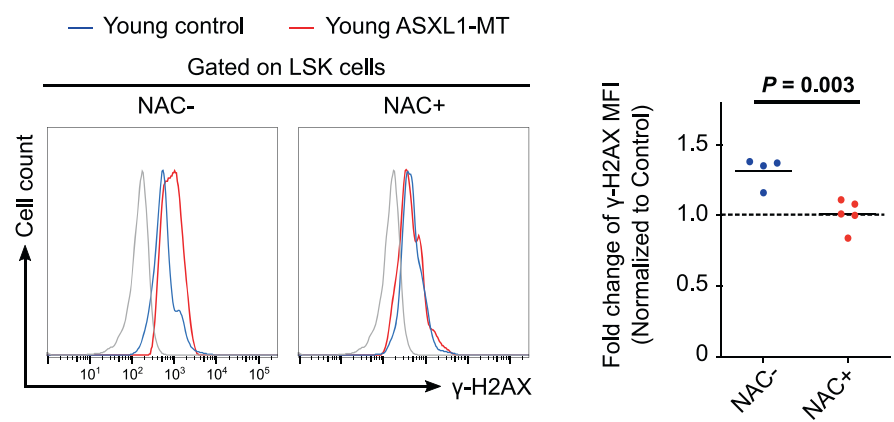

d

f

g
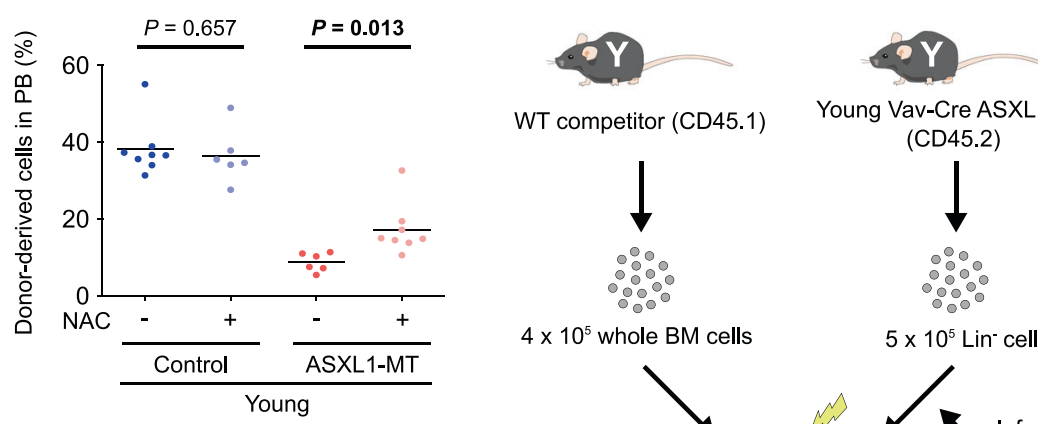

$\rightarrow$ Mock $\rightarrow$ Catalase/SOD2 (CD45.2)

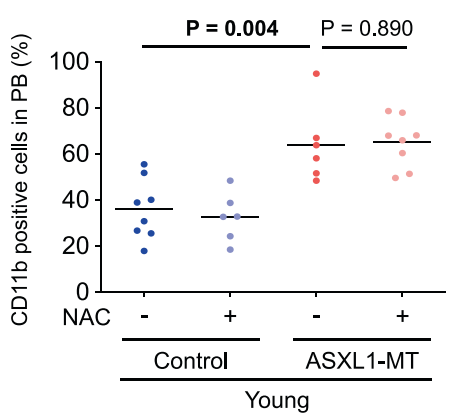

$4 \times 10^{5}$ whole BM cells

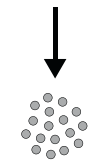

$5 \times 10^{5}$ Lin $^{-}$cells

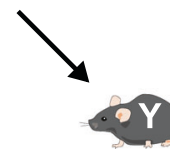

Lethally irradiated WT recipient (CD45.1)

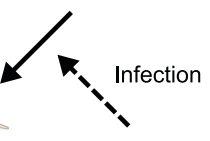

SF91-IRES-Venus (Mock)

or

SF91-Cat/SOD2-IRES-Venus

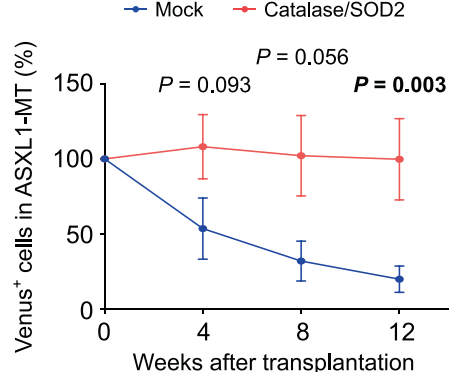

PB chimerism analysis

Fig. 8 ROS-mediated DNA damage causes dysfunction of LT-HSCs. a The experimental design for transplantation using whole bone marrow cells treated with an antioxidant scavenger NAC. Whole bone marrow cells isolated from young control and young Vav-Cre ASXL1-MT KI mice that had been treated with NAC for 8 weeks were transplanted into lethally irradiated recipient mice with competitor cells. Recipient mice were continuously treated with NAC after transplantation. b, c 6 weeks after transplantation, effects of NAC administration on ROS (b) and $\gamma$-H2AX levels (c) in LSK cells were determined $(n=4(N A C-)$ and $5(N A C+))$. d, e The frequencies of donor-derived cells $(\mathbf{d})$ and CD11b-positive cells in donor-derived cells $(\mathbf{e})$ in peripheral blood were analyzed $(n=8$ (Control-NAC-), 6 (Control-NAC+), 6 (ASXL1-MT-NAC-), and 8 (ASXL1-MT-NAC+)). f The experimental design for transplantations using Lin- cells transduced with ROS-detoxifying enzymes. Lin ${ }^{-}$cells from young Vav-Cre ASXL1-MT KI mice transduced with control or Catalase-SOD2 retrovirus were transplanted into lethally irradiated recipient mice. $\mathbf{g}$ Engraftment was assessed at the indicated weeks after transplantation $(n=7$ (Mock) and 8 (Catalase-SOD2)). Data are mean \pm s.e.m. Statistical significances are assessed by two-tailed Student's $t$-test. ${ }^{\star} P \leq 0.05,{ }^{\star \star} P \leq 0.01$. 
a

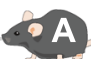

Aged control or Vav-Cre ASXL1-MT

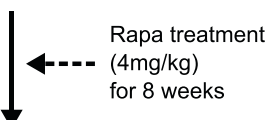

BM analysis

- Cell count

- Mitochondrial membrane potential

(MitoTracker)

- Intracellular ROS (CellROX)

d

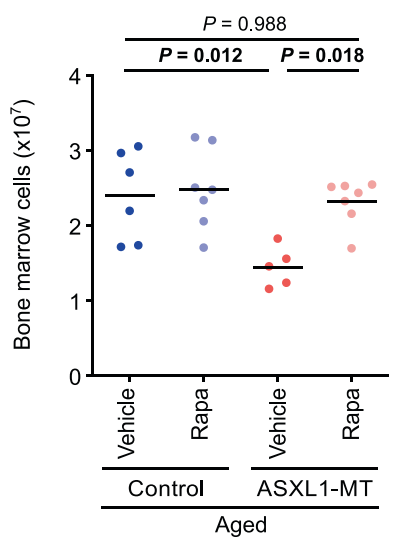

b

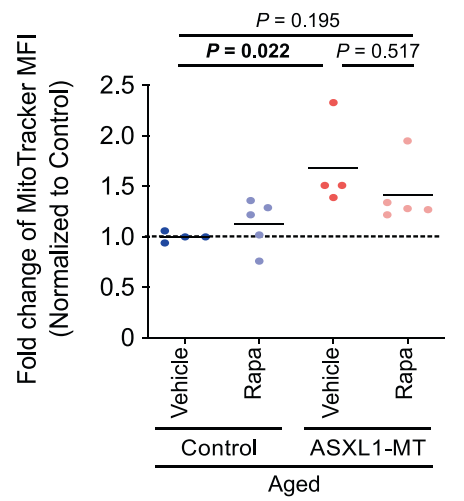

e

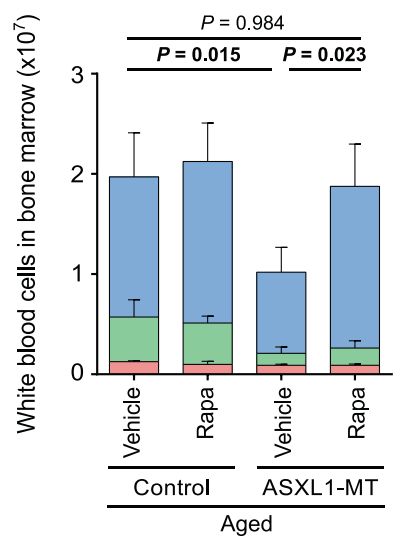

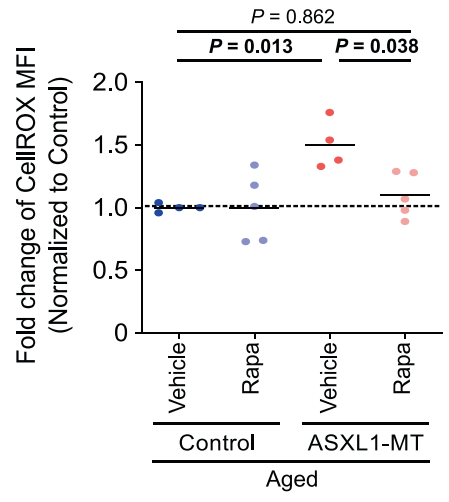

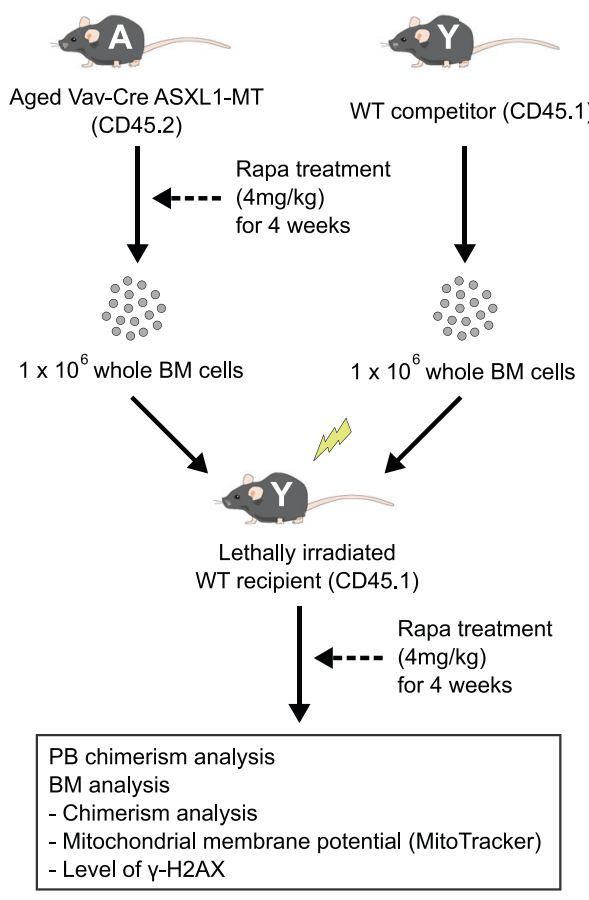

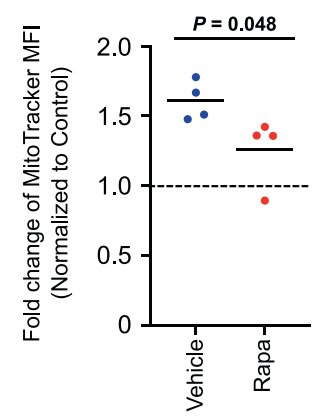

i

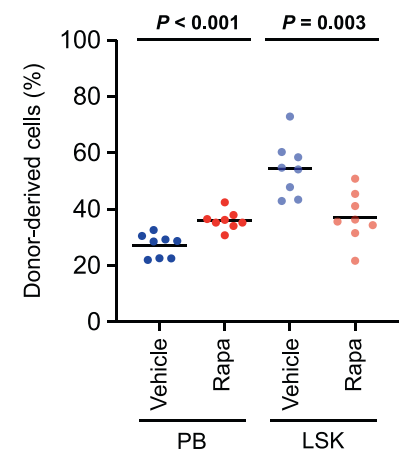

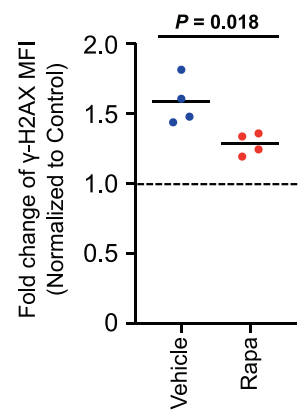

j

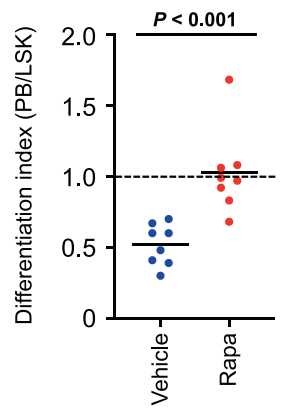

sequenced on the Illumina HiSeq2500 System with $2 \times 100$ nucleotide paired-end reads according to the manufacturer's protocol. Derived reads were processed using cutadapt (1.8.1) and fastx-toolkit (0.0.13) to remove Illumina adaptor sequence and to trim low-quality bases. Quality of reads was assessed using FastQC. Processed reads were aligned to GRCm 38 reference transcripts using TopHat (2.1.1) - Cufflinks (2.1.1) pipeline to derive gene FPKM values ${ }^{73-75}$.
Gene set enrichment analysis (GSEA) was performed using the GSEA tool from the Broad Institute (http://software.broadinstitute.org/gsea/). Heat maps were generated using heatmap.2 function of gplots package (3.0.4), from the Bioconductor. The microarray data for HSCs of young and aged wild-type mice are publically available at Gene Expression Omnibus (GEO) with the reference series tag "GSE 48893". 
Fig. 9 The dysregulated hematopoiesis in aged ASXL1-MT KI mice is reverted by treatment with rapamycin. a Aged Vav-Cre ASXL1-MT KI mice and age-matched control mice were treated with rapamycin intraperitoneally every other day for 8 weeks. At the end of administrations, end-point analyses of bone marrow cells were conducted. b, c Mitochondrial membrane potential (b) and intracellular ROS levels (c) in LT-HSCs were analyzed ( $n=4$ (ControlVehicle), 5 (Control-Rapamycin), 4 (ASXL1-MT-Vehicle), and 5 (ASXL1-MT-Rapamycin)). Results are expressed as fold change relative to vehicle control group. $\mathbf{d}$ Absolute numbers of bone marrow cells per leg ( $n=6$ (Control-Vehicle), 7 (Control-Rapamycin), 5 (ASXL1-MT-Vehicle), and 7 (ASXL1-MTRapamycin)). e Absolute numbers of white blood cells in bone marrow per leg ( $n=5$ (Control-Vehicle), 6 (Control-Rapamycin), 5 (ASXL1-MT-Vehicle), and 6 (ASXL1-MT-Rapamycin)). Data are mean \pm s.d. $\mathbf{f}$ The experimental design for transplantation using whole bone marrow cells treated with rapamycin. Whole bone marrow cells isolated from aged Vav-Cre ASXL1-MT KI mice and age-matched control mice that had been treated with rapamycin for 4 weeks were transplanted into lethally irradiated recipient mice with competitor cells. Recipient mice were continuously treated with rapamycin after transplantation. $\mathbf{g}$, $\mathbf{h} 4$ weeks after transplantation, effects of rapamycin treatment on mitochondrial membrane potential $(\mathbf{g})$ and $\gamma$ - $\mathrm{H} 2 \mathrm{AX}$ levels $(\mathbf{h})$ in LSK cells were determined $(n=4)$. $\mathbf{i}, \mathbf{j} 4$ weeks after transplantation, the frequency of donor-derived cells in peripheral blood and LSK cells were analyzed $(n=8)(\mathbf{i})$. Differentiation index, which is obtained by dividing the frequency of donor-derived cells in peripheral blood by LSK cells, was calculated (j). Statistical significances are assessed by one-way ANOVA with Tukey-Kramer's post-hoc test $(\mathbf{b}-\mathbf{e})$ or two-tailed Student's $t$-test $\left(\mathbf{g}\right.$-j). ${ }^{\star} P \leq 0.05$,

${ }^{\star \star} P \leq 0.01,{ }^{\star \star \star} P \leq 0.001$

a
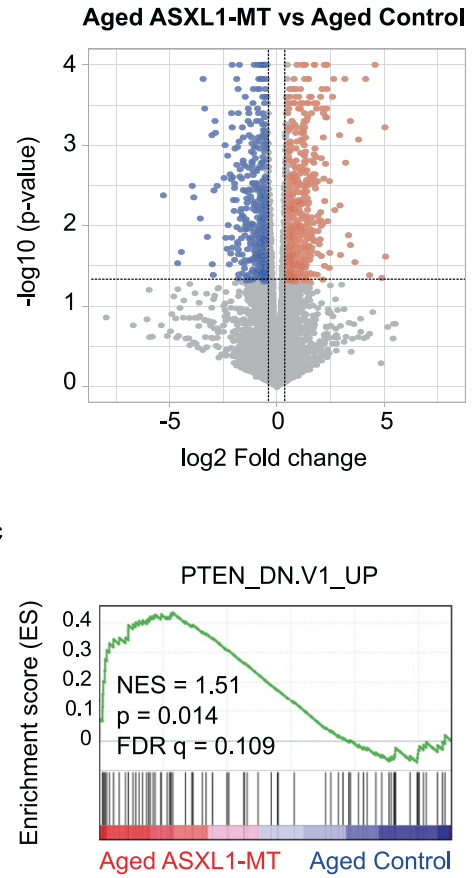

b

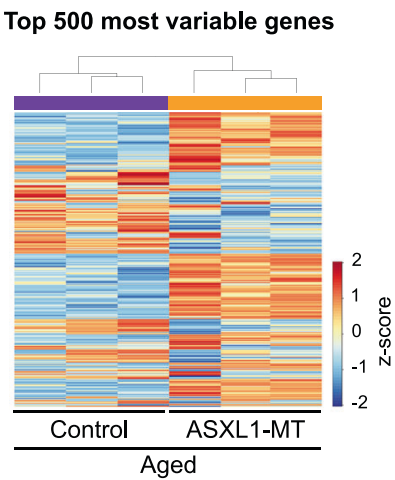

d

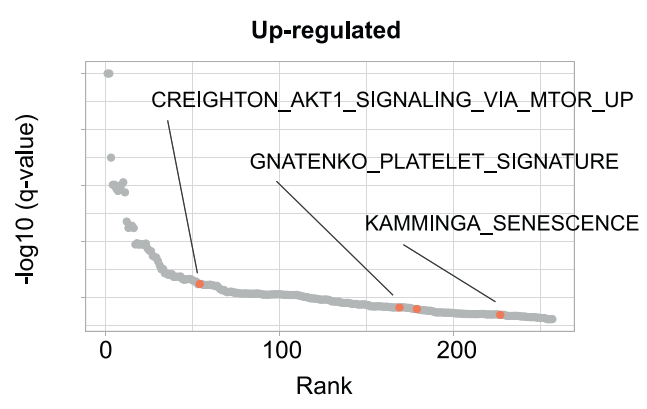

Down-regulated
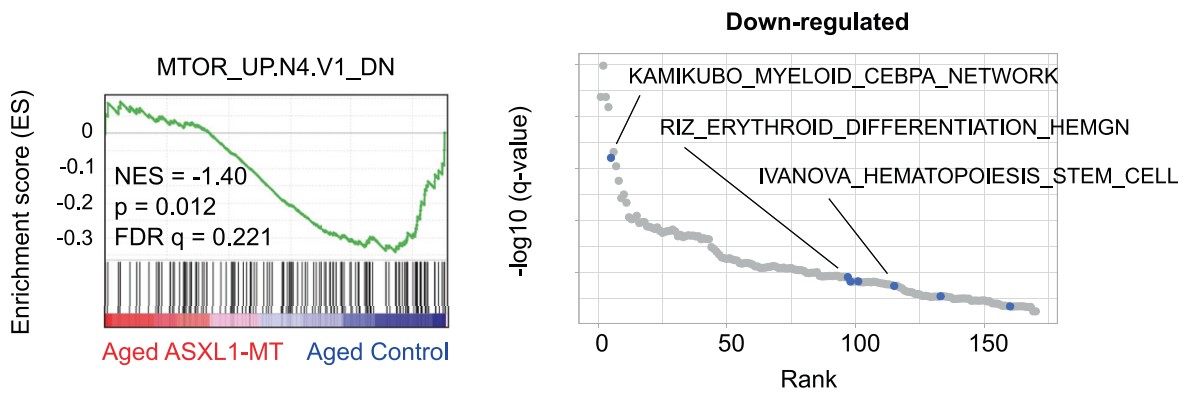

f

e

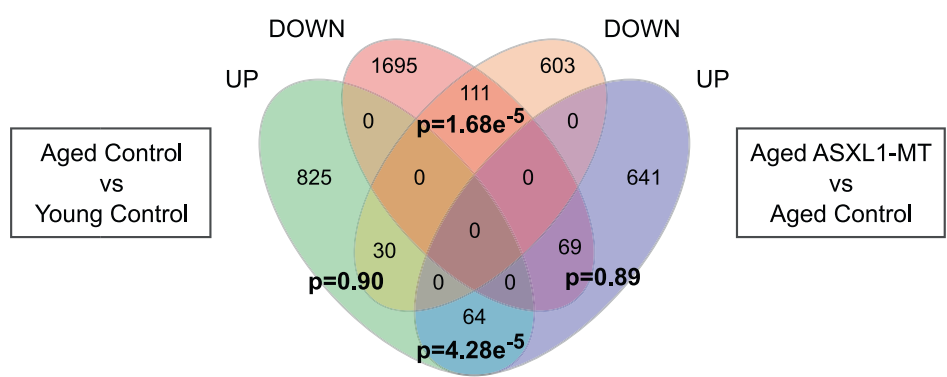

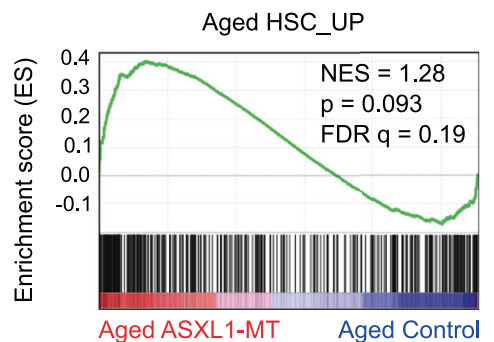

Fig. 10 ASXL1-MT enforces age-associated gene expression patterns. a Volcano plot displaying differentially expressed genes in LT-HSCs between aged Vav-Cre ASXL1-MT KI mice and aged-matched control mice $(n=3)$. b The heatmap of the top 500 most variable genes $(n=3)$. c GSEA using genesets upregulated by PTEN deficiency (PTEN_DN.V1_UP) and negatively regulated by mTOR signaling (MTOR_UP.N4.V1_DN). d Up-regulated or down-regulated genesets (MSigDB C2_CGP: chemical and genetic perturbations) in LT-HSCs from aged Vav-Cre ASXL1-MT KI mice. Up-regulated genesets involved in $\mathrm{Akt} / \mathrm{mTOR}$ signaling, platelet signature, and senescence signature are shown with orange circles. Down-regulated genesets involved in myeloid differenciation, erythroid differentiation, and HSC signature are shown with blue circles. e Venn diagrams showing up-regulated or down-regulated genes in LT-HSCs of (1) aged Vav-Cre ASXL1-MT KI mice compared to aged control mice and (2) aged wild-type mice compared to young wild-type mice (GSE48893). Statistical significances are assessed by two-sided Fisher's exact test. f GSEA using a geneset up-regulated in LT-HSCs with age (GSE48893). 
Comet assay. Alkaline comet assays were performed with CometAssay Kit (Trevigen). Sort-purified LT-HSCs were embedded in LM Agarose and transferred onto Comet Slides. Cells were then lysed with lysis solution and soaked in alkaline solution (200 mM NaOH, $1 \mathrm{mM}$ EDTA). Comet Slides were subjected to electrophoresis in alkaline solution followed by staining with SYBR Green. Analysis was performed using the ImageJ software.

Single cell liquid culture. Freshly isolated LT-HSCs were single cell sorted into 96well round-bottom plates and were cultured in IMDM supplemented with $20 \%$ FBS, $1 \%$ penicillin/streptomycin, $2 \mathrm{mM}$ L-glutamine, $50 \mathrm{mM}$ 2-Mercaptoethanol (Thermo Fisher Scientific; $21985-023)$, SCF (50 ng/mL), TPO (50 ng/mL), IL-3 (10 ng/mL), IL-6 (10 ng/mL) for 2 weeks.

Subcellular fractionation. To isolate the nucleus, cells were washed with ice-cold PBS, harvested with hypotonic buffer (10 mM HEPES pH 7.0, $1.5 \mathrm{mM} \mathrm{MgCl}_{2}$, $10 \mathrm{mM} \mathrm{KCl}, 0.5 \mathrm{mM}$ DTT, $0.1 \%$ TritonX-100, $2 \mathrm{mM} \mathrm{Na}_{3} \mathrm{VO}_{4}, 2 \mathrm{mM}$ PMSF, 50 $\mathrm{mM} \mathrm{NaF}, 10 \mu \mathrm{M}$ MG132) supplemented with proteinase inhibitor (cOmplete Mini, Roche, 11836153001), and subsequently incubated for $20 \mathrm{~min}$ on ice. Lysates were centrifuged at $3500 \times g$ for 5 min and subsequently separated into supernatants (cytoplasmic fraction) and pellets (nuclear fraction). The pellets were lysed with lysis buffer (TBS, 0.5\% NP-40, $2 \mathrm{mM} \mathrm{Na} \mathrm{VO}_{4}, 2 \mathrm{mM}$ PMSF, $50 \mathrm{mM} \mathrm{NaF}, 10 \mu \mathrm{M}$ MG132, proteinase inhibitor cocktail) followed by incubation for $30 \mathrm{~min}$ on ice with pipetting occasionally. Lysates were then centrifuged at $11,000 \times g$ for $30 \mathrm{~min}$ at $4{ }^{\circ} \mathrm{C}$ and supernatants were collected as a nuclear fraction.

Coimmunoprecipitation and western blotting. 293T cells were transfected with plasmids using PEI. $48 \mathrm{~h}$ after transfection, cells were washed with ice-cold PBS and were lysed with cell lysis buffer (50 mM Tris- $\mathrm{HCl}, 1 \mathrm{mM}$ EDTA, $150 \mathrm{mM} \mathrm{NaCl}, 1 \%$ TritonX-100, 0.1\% SDS, $2 \mathrm{mM} \mathrm{Na}_{3} \mathrm{VO}_{4}, 2 \mathrm{mM}$ PMSF, $50 \mathrm{mM} \mathrm{NaF}, 10 \mu \mathrm{M}$ MG132) supplemented with proteinase inhibitor (cOmplete Mini, Roche, 11836153001). Lysates are immunoprecipitated with anti-FLAG (Sigma, F1804, 1:100) or antiAKT1/2/3 (Santa Cruz Biotechnology, sc-81434, 1:20) antibody using Dynabeads Protein G (Thermo Fisher Scientific, 10004D) for $10 \mathrm{~min}$ at room temperature. Immunoprecipitated samples were subjected to electrophoresis, transfer to nitrocellulose membranes, and blocking with $5 \%$ bovine serum albumin (BSA)/TBS for $1 \mathrm{~h}$ at room temperature. Samples were then incubated with primary antibodies in $1 \%$ BSA $/ 0.1 \%$ Tween $20 / \mathrm{TBS}$ overnight at $4{ }^{\circ} \mathrm{C}$, washed and incubated with secondary antibodies in $1 \%$ BSA/0.1\% Tween 20/TBS for $1 \mathrm{~h}$ at room temperature. The primary antibodies used in immunoblotting are as follows: anti-AKT1/2/3 (Santa Cruz Biotechnology, sc-81434, 1:200), anti-Akt (Pan) (Cell Signaling Technology, \#4691, 1:1000), anti-Phospho-Akt (Ser473) (Cell signaling Technology, \#4060, 1:2000), anti-BAP1 (Santa Cruz Biotechnology, sc-28383, 1:200), antiFLAG (Sigma, F1804, 1:1000), anti-GAPDH (Cell signaling Technology, \#5174, 1:2000), anti-Myc (Roche, \#11814150001, 1:1000), anti-HA (Roche, \#11867423001, 1:1000), anti- $\alpha$-Tubulin (SIGMA, T9026, 1:3000), and anti-Lamin B1 (Santa Cruz Biotechnology, sc-374015, 1:50).

Human data analysis. The clinical microarray data for CD34-positive bone marrow cells from 159 MDS patients, including 13 patients with DNMT3A mutations, 33 patients with TET2 mutations, and 21 patients with ASXL1 mutations, and 17 healthy controls are publically available at Gene Expression Omnibus (GEO) with the reference series tag "GSE58831".

Statistics and reproducibility. Data are expressed as mean \pm standard deviation (s.d.) or standard error of mean (s.e.m.). $P$ values were calculated using unpaired, two-tailed Student's $t$-test, Mann-Whitney rank sum test, one-way ANOVA with Tukey-Kramer's post-hoc test, or two-sided Fisher's exact test as indicated in figure legend. The significance between the survival of cohorts was assessed by Kaplan-Meier survival analysis and log-ranked tests. $P$ values $<0.05$ were considered significant. All calculations were performed by using GraphPad Prism software. Sample size ' $n$ ' indicates biological replicates. No randomization or blinding was used and no animals were excluded from the analysis.

Reporting summary. Further information on research design is available in the Nature Research Reporting Summary linked to this article.

\section{Data availability}

RNA-seq data are available in sequence read archive (SRA) database (accession ID: PRJNA673672). Metabolome analysis data are available in MassIVE (accession ID: MSV000086816). The datasets generated or analyzed during the current study are available from the corresponding author (kitamura@ims.u-tokyo.ac.jp) on reasonable request. Source data are provided with this paper.

Received: 5 December 2019; Accepted: 23 February 2021; Published online: 23 March 2021

\section{References}

1. De Haan, G. \& Lazare, S. S. Aging of hematopoietic stem cells. Blood 131, 479-488 (2018).

2. Akunuru, S. \& Geiger, H. Aging, clonality, and rejuvenation of hematopoietic stem cells. Trends Mol. Med. 22, 701-712 (2016).

3. Geiger, H., De Haan, G. \& Carolina Florian, M. The ageing haematopoietic stem cell compartment. Nat. Rev. Immunol. 13, 376-389 (2013).

4. De Haan, G., Nijhof, W. \& Zant, G. Van Mouse strain-dependent changes in frequency and proliferation of hematopoietic stem cells during aging: correlation between lifespan and cycling activity. Blood 89, 1543-1550 (1997).

5. Sudo, K., Ema, H., Morita, Y. \& Nakauchi, H. Age-associated characteristics of murine hematopoietic stem cells. J. Exp. Med. 192, 1273-1280 (2000).

6. Beerman, I. et al. Functionally distinct hematopoietic stem cells modulate hematopoietic lineage potential during aging by a mechanism of clonal expansion. Proc. Natl Acad. Sci. USA 107, 5465-5470 (2010).

7. Dykstra, B., Olthof, S., Schreuder, J., Ritsema, M. \& de Haan, G. Clonal analysis reveals multiple functional defects of aged murine hematopoietic stem cells. J. Exp. Med. 208, 2691-2703 (2011)

8. Bernitz, J. M., Kim, H. S., Macarthur, B., Sieburg, H. \& Moore, K. Hematopoietic stem cells count and remember article hematopoietic stem cells count and remember self-renewal divisions. Cell 167, 1296-1309 (2016).

9. Yamamoto, R. et al. Large-scale clonal analysis resolves aging of the mouse hematopoietic stem cell compartment. Cell Stem Cell 22, 600-607 (2018).

10. Fisher, C. L., Berger, J., Randazzo, F. \& Brock, H. W. A human homolog of additional sex combs, ADDITIONAL SEX COMBS-LIKE 1, maps to chromosome 20q11. Gene 306, 115-126 (2003).

11. Abdel-Wahab, O. et al. ASXL1 mutations promote myeloid transformation through loss of PRC2-mediated gene repression. Cancer Cell 22, 180-193 (2012).

12. Scheuermann, J. C. et al. Histone $\mathrm{H} 2 \mathrm{~A}$ deubiquitinase activity of the Polycomb repressive complex PR-DUB. Nature 465, 243-247 (2010).

13. Inoue, D. et al. A novel ASXL1-OGT axis plays roles in $\mathrm{H} 3 \mathrm{~K} 4$ methylation and tumor suppression in myeloid malignancies. Leukemia 32, 1327-1337 (2018).

14. Thol, F. et al. Prognostic significance of ASXL1 mutations in patients with myelodysplastic syndromes. J. Clin. Oncol. 29, 2499-2506 (2011).

15. Gelsi-Boyer, V. et al. ASXL1 mutation is associated with poor prognosis and acute transformation in chronic myelomonocytic leukaemia. Br. J. Haematol. 151, 365-375 (2010).

16. Nangalia, J. et al. Somatic CALR mutations in myeloproliferative neoplasms with nonmutated JAK2. N. Engl. J. Med. 369, 2391-2405 (2013).

17. Schnittger, S. et al. ASXL1 exon 12 mutations are frequent in AML with intermediate risk karyotype and are independently associated with an adverse outcome. Leukemia 27, 82-91 (2013).

18. Boultwood, J. et al. Frequent mutation of the polycomb-associated gene ASXL1 in the myelodysplastic syndromes and in acute myeloid leukemia. Leukemia 24, 1062-1065 (2010).

19. Xie, M. et al. Age-related mutations associated with clonal hematopoietic expansion and malignancies. Nat. Med. 20, 1472-1478 (2014).

20. Genovese, G. et al. Clonal hematopoiesis and blood-cancer risk inferred from blood DNA sequence. N. Engl. J. Med. 371, 2477-2487 (2014).

21. Jaiswal, S. et al. Age-related clonal hematopoiesis associated with adverse outcomes. N. Engl. J. Med. 371, 2488-2498 (2014).

22. Inoue, D. et al. Truncation mutants of ASXL1 observed in myeloid malignancies are expressed at detectable protein levels. Exp. Hematol. 44, 172-176 (2016).

23. Asada, S. et al. Mutant ASXL1 cooperates with BAP1 to promote myeloid leukaemogenesis. Nat. Commun. 9, 1-18 (2018).

24. Balasubramani, A. et al. Cancer-associated ASXL1 mutations may act as gainof-function mutations of the ASXL1-BAP1 complex. Nat. Commun. 6, 1-15 (2015).

25. Nagase, R. et al. Expression of mutant Asxll perturbs hematopoiesis and promotes susceptibility to leukemic transformation. J. Exp. Med. 215, 1729-1747 (2018).

26. Bowman, R. L., Busque, L. \& Levine, R. L. Clonal hematopoiesis and evolution to hematopoietic malignancies. Cell Stem Cell 22, 157-170 (2018).

27. Shlush, L. I. Age-related clonal hematopoiesis. Blood 131, 496-504 (2018).

28. Sperling, A. S., Gibson, C. J. \& Ebert, B. L. The genetics of myelodysplastic syndrome: from clonal haematopoiesis to secondary leukaemia. Nat. Rev. Cancer 17, 5-19 (2017).

29. Ferrando, A. A. \& López-Otín, C. Clonal evolution in leukemia. Nat. Med. 23, 1135-1145 (2017).

30. Quivoron, C. et al. TET2 inactivation results in pleiotropic hematopoietic abnormalities in mouse and is a recurrent event during human lymphomagenesis. Cancer Cell 20, 25-38 (2011).

31. Moran-Crusio, K. et al. Tet2 loss leads to increased hematopoietic stem cell self-renewal and myeloid transformation. Cancer Cell 20, 11-24 (2011). 
32. Challen, G. A. et al. Dnmt3a is essential for hematopoietic stem cell differentiation. Nat. Genet. 44, 23-31 (2012).

33. Challen, G. A. et al. Dnmt3a and Dnmt3b have overlapping and distinct functions in hematopoietic stem cells. Cell Stem Cell 15, 350-364 (2014).

34. Uni, M. et al. Modeling ASXL1 mutation revealed impaired hematopoiesis caused by derepression of p16Ink4a through aberrant PRC1-mediated histone modi fi cation. Leukemia 191-204 (2018) https://doi.org/10.1038/s41375-0180198-6

35. Hsu, Y. C. et al. The distinct biological implications of Asxl1 mutation and its roles in leukemogenesis revealed by a knock-in mouse model. J. Hematol. Oncol. 10, 1-15 (2017).

36. Gekas, C. \& Graf, T. CD41 expression marks myeloid-biased adult hematopoietic stem cells and increases with age. Blood 121, 4463-4472 (2013).

37. Yamamoto, R. et al. Clonal analysis unveils self-renewing lineage-restricted progenitors generated directly from hematopoietic stem cells. Cell 154, 1112-1126 (2013).

38. Bae, S. et al. Akt is negatively regulated by the MULAN E3 ligase. Cell Res. 22, 873-885 (2012).

39. Dickey, C. A. et al. Akt and CHIP coregulate tau degradation through coordinated interactions. Proc. Natl Acad. Sci. USA 105, 3622-3627 (2008).

40. Fan, C. D., Lum, M. A., Xu, C., Black, J. D. \& Wang, X. Ubiquitin-dependent regulation of phospho-AKT dynamics by the ubiquitin E3 LIGASE, NEDD41 , in the insulin-like growth factor-1 response. J. Biol. Chem. 288, 1674-1684 (2013).

41. Suizu, F. et al. The E3 ligase TTC3 facilitates ubiquitination and degradation of phosphorylated Akt. Dev. Cell 17, 800-810 (2009).

42. Xiang, T. et al. Negative regulation of AKT activation by BRCA1. Cancer Res. 68, 10040-10044 (2008).

43. Wang, J. et al. Protein interaction data set highlighted with human RasMAPK/PI3K signaling pathways research articles. J. Proteome Res. 7, 3879-3889 (2008).

44. Youn, H. S. et al. Asxll deficiency in embryonic fibroblasts leads to cellular senescence via impairment of the AKT-E2F pathway and Ezh2 inactivation. Sci. Rep. 7, 1-13 (2017).

45. Inoue, D. et al. SETBP1 mutations drive leukemic transformation in ASXL1mutated MDS. Leukemia 29, 847-857 (2015).

46. Chen, C. et al. TSC-mTOR maintains quiescence and function of hematopoietic stem cells by repressing mitochondrial biogenesis and reactive oxygen species. J. Exp. Med. 205, 2397-2408 (2008).

47. Luchsinger, L. L., De Almeida, M. J., Corrigan, D. J., Mumau, M. \& Snoeck, H. W. Mitofusin 2 maintains haematopoietic stem cells with extensive lymphoid potential. Nature 529, 528-531 (2016).

48. Yu, W. M. et al. Metabolic regulation by the mitochondrial phosphatase PTPMT1 is required for hematopoietic stem cell differentiation. Cell Stem Cell 12, 62-74 (2013)

49. Archer, S. L. Mitochondrial dynamics-mitochondrial fission and fusion in human diseases. N. Engl. J. Med. 369, 2236-2251 (2013).

50. Westermann, B. Mitochondrial fusion and fission in cell life and death. Nat. Rev. Mol. Cell Biol. 11, 872-884 (2010).

51. Yahata, T. et al. Accumulation of oxidative DNA damage restricts the selfrenewal capacity of human hematopoietic stem cells. Blood 118, 2941-2951 (2015).

52. Douglas, C. W. Mitochondria and cancer. Nat. Rev. Cancer 12, 685-698 (2012).

53. Abbas, H. A. et al. Mdm2 is required for survival of hematopoietic stem cells/ progenitors via dampening of ROS-induced p53 activity. Cell Stem Cell 7, 606-617 (2010).

54. Sablina, A. A. et al. The antioxidant function of the p53 tumor suppressor. Nat. Med. 11, 1306-1313 (2005).

55. Dumble, M. et al. The impact of altered p53 dosage on hematopoietic stem cell dynamics during aging. Blood 109, 1736-1742 (2007).

56. Cunningham, J. T. et al. mTOR controls mitochondrial oxidative function through a YY1-PGC-1a transcriptional complex. Nature 450, 736-740 (2007).

57. Ma, L., Chen, Z., Erdjument-Bromage, H., Tempst, P. \& Pandolfi, P. P. Phosphorylation and functional inactivation of TSC2 by Erk: implications for tuberous sclerosis and cancer pathogenesis. Cell 121, 179-193 (2005).

58. Yilmaz, Ö. H. et al. Pten dependence distinguishes haematopoietic stem cells from leukaemia-initiating cells. Nature 441, 475-482 (2006).

59. Zhang, J. et al. PTEN maintains haematopoietic stem cells and acts in lineage choice and leukaemia prevention. Nature 441, 518-522 (2006).

60. Bononi, A. et al. BAP1 regulates IP3R3-mediated Ca2+ flux to mitochondria suppressing cell transformation. Nature 546, 549-553 (2017).

61. Machida, Y. J., Machida, Y., Vashisht, A. A., Wohlschlegel, J. A. \& Dutta, A. The deubiquitinating enzyme BAP1 regulates cell growth via interaction with HCF-1. J. Biol. Chem. 284, 34179-34188 (2009).

62. Misaghi, S. et al. Association of C-terminal ubiquitin hydrolase BRCA1associated protein 1 with cell cycle regulator host cell factor 1. Mol. Cell. Biol. 29, 2181-2192 (2009).
63. Qin, J. et al. BAP1 promotes breast cancer cell proliferation and metastasis by deubiquitinating KLF5. Nat. Commun. 6, 8471 (2015).

64. Ruan, H. Bin et al. O-GlcNAc transferase/host cell factor $\mathrm{C} 1$ complex regulates gluconeogenesis by modulating PGC-1a stability. Cell Metab. 16, 226-237 (2012)

65. Cao, L. et al. Deregulation of tumor suppressive ASXL1-PTEN/AKT axis in myeloid malignancies. J. Mol. Cell Biol. 12, 688-699 (2020).

66. Jaiswal, S. et al. Clonal hematopoiesis and risk of atherosclerotic cardiovascular disease. N. Engl. J. Med. 377, 111-121 (2017).

67. Yizhak, K. et al. RNA sequence analysis reveals macroscopic somatic clonal expansion across normal tissues. Science (80-.). 364, eaaw0726 (2019).

68. Tamura, M. et al. Opposing effects of acute versus chronic inhibition of p53 on decitabine's efficacy in myeloid neoplasms. Sci. Rep. 9, 4-6 (2019).

69. Rehman, J. et al. Inhibition of mitochondrial fission prevents cell cycle progression in lung cancer. FASEB J. 26, 2175-2186 (2012).

70. Senyilmaz, D. et al. Regulation of mitochondrial morphology and function by stearoylation of TFR1. Nature 525, 124-128 (2015).

71. Kunisawa, J. et al. Mode of bioenergetic metabolism during B cell differentiation in the intestine determines the distinct requirement for vitamin B1. Cell Rep. 13, 122-131 (2015).

72. Miyajima, M. et al. Metabolic shift induced by systemic activation of T cells in PD-1-deficient mice perturbs brain monoamines and emotional behavior. Nat. Immunol. 18, 1342-1352 (2017).

73. Dobin, A. \& Gingeras, T. R. “TopHat2: accurate alignment of transcriptomes in the presence of insertions, deletions and gene fusions. Genome Biol. 14, R36 (2013).

74. Trapnell, C. et al. Differential gene and transcript expression analysis of RNA-seq experiments with TopHat and Cufflinks. Nat. Protoc. 7, 562-578 (2012).

75. Trapnell, C. et al. Transcript assembly and quantification by RNA-Seq reveals unannotated transcripts and isoform switching during cell differentiation. Nat. Biotechnol. 28, 511-515 (2010).

\section{Acknowledgements}

We thank K. Matsuda for sharing $p 53^{-1-}$ mice, Michael D. Milsom for providing SF91IRES-GFP and SF91-Cat/SOD2-IRES-GFP vectors, IMSUT FACS Core Laboratory for flow cytometry and Nikon Imaging Laboratory for microscopic analysis. This work was supported by Grant-in-Aid for Scientific Research (B) (No. 15H04855) (to T.K.), Grantin-Aid for Scientific Research on Innovative Areas "Stem Cell Aging and Disease" (No. 17H05634), The Tokyo Biochemical Research Foundation (to T.K.), Japanese Society of Hematology (to T.K.), The Japan foundation for Aging and Health (to S.G.), Suzuken Memorial Foundation (to S.G.). Infrastructure of metabolomics was partly supported by JST ERATO Suematsu Gas Biology (2010-2015) (to Y.S. and M.S.). O.A.W. is supported by grants from a Leukemia \& Lymphoma Society Specialized Center for Research award as well as funding from the Edward P. Evans MDS Foundation and the Henry \& Marilun Taub Foundation.

\section{Author contributions}

Ta.F. designed and performed experiments and analyzed data with assistance from S.A A.T., S.S., N.S., To.F., Y.T., Ts.F, R.T., and K.Y. Y.S., and M.S. performed metabolome analysis. S.Y., A.M., K.Y., Y.I., Y.F., and T.S. performed RNA-seq analysis. D.I., H.M., E.K.N., and O.A.-W. provided critical materials, knowledge on their use, and concepts. H.H. generated ASXL1-MT cKI mice. S.G. and T.K. guided research and wrote the paper with Ta.F.

\section{Competing interests}

O.A.-W. has served as a consultant for H3B Biomedicine, Foundation Medicine Inc., Merck, and Janssen and serves on the scientific advisory board for Envisagenics Inc.; O.A.-W. has received personal speaking fees from Daiichi Sankyo. Other authors declare no competing interests.

\section{Additional information}

Supplementary information The online version contains supplementary material available at https://doi.org/10.1038/s41467-021-22053-y.

Correspondence and requests for materials should be addressed to T.K.

Peer review information Nature Communications thanks Yan Liu and the other, anonymous, reviewer(s) for their contribution to the peer review of this work. Peer reviewer reports are available.

Reprints and permission information is available at http://www.nature.com/reprints

Publisher's note Springer Nature remains neutral with regard to jurisdictional claims in published maps and institutional affiliations. 
(c) (i) Open Access This article is licensed under a Creative Commons Attribution 4.0 International License, which permits use, sharing, adaptation, distribution and reproduction in any medium or format, as long as you give appropriate credit to the original author(s) and the source, provide a link to the Creative Commons license, and indicate if changes were made. The images or other third party material in this article are included in the article's Creative Commons license, unless indicated otherwise in a credit line to the material. If material is not included in the article's Creative Commons license and your intended use is not permitted by statutory regulation or exceeds the permitted use, you will need to obtain permission directly from the copyright holder. To view a copy of this license, visit http://creativecommons.org/ licenses/by/4.0/.

(C) The Author(s) 2021 